\title{
WestVirginiaUniversity
}

THE RESEARCH REPOSITORY @ WVU

Graduate Theses, Dissertations, and Problem Reports

2005

\section{Multi-resolution dental image registration based on genetic algorithm}

\author{
Mythili Ogirala \\ West Virginia University
}

Follow this and additional works at: https://researchrepository.wvu.edu/etd

\section{Recommended Citation}

Ogirala, Mythili, "Multi-resolution dental image registration based on genetic algorithm" (2005). Graduate Theses, Dissertations, and Problem Reports. 1610.

https://researchrepository.wvu.edu/etd/1610

This Thesis is protected by copyright and/or related rights. It has been brought to you by the The Research Repository @ WVU with permission from the rights-holder(s). You are free to use this Thesis in any way that is permitted by the copyright and related rights legislation that applies to your use. For other uses you must obtain permission from the rights-holder(s) directly, unless additional rights are indicated by a Creative Commons license in the record and/ or on the work itself. This Thesis has been accepted for inclusion in WVU Graduate Theses, Dissertations, and Problem Reports collection by an authorized administrator of The Research Repository @ WVU. For more information, please contact researchrepository@mail.wvu.edu. 


\title{
MULTI RESOLUTION DENTAL IMAGE REGISTRATION BASED ON GENETIC ALGORITHM
}

Mythili Ogirala

\author{
Thesis submitted to the \\ College of Engineering and Mineral Resources \\ at West Virginia University \\ in partial fulfillment of the requirements \\ for the degree of \\ Master of Science \\ in \\ Electrical Engineering
}

Dr. Hany Ammar, Ph.D., Chair

Dr. Xin Li, Ph.D.

Dr. Gamal Fahmy, Ph.D.

Lane Department of Computer Science and Electrical Engineering Morgantown, West Virginia

2005

Keywords: ADIS, Dental Forensics, Image Registration, Multi resolutions, Genetic algorithms, Similarity measure, Affine transformations

Copyright 2005 Mythili Ogirala 


\section{ABSTRACT \\ MULTI RESOLUTION DENTAL IMAGE REGISTRATION BASED ON GENETIC ALGORITHM}

\section{Mythili Ogirala}

The Automated Dental Identification System (ADIS) is a Post Mortem Dental Identification System. This thesis presents dental image registration, required for the preprocessing steps of the image comparison component of ADIS. We proposed a multi resolution dental image registration based on genetic algorithms. The main objective of this research is to develop techniques for registration of extracted subject regions of interest with corresponding reference regions of interest.

We investigated and implemented registration using two multi resolution techniques namely image sub sampling and wavelet decomposition. Multi resolution techniques help in the reduction of search data since initial registration is carried at lower levels and results are updated as the levels of resolutions increase. We adopted edges as image features that needed to be aligned. Affine transformations were selected to transform the subject dental region of interest to achieve better alignment with the reference region of interest. These transformations are known to capture complex image distortions. The similarity between subject and reference image has been computed using Oriented Hausdorff Similarity measure that is robust to severe noise and image degradations. A genetic algorithm was adopted to search for the best transformation parameters that give maximum similarity score.

Testing results show that the developed registration algorithm yielded reasonable results in accuracy for dental test cases that contained slight misalignments. The relative percentage errors between the known and estimated transformation parameters were less than $20 \%$ with a termination criterion of a ten minute time limit. Further research is needed for dental cases that contain high degree of misalignment, noise and distortions. 


\section{ACKNOWLEDGEMENTS}

To begin with I have been very fortunate to work in the ADIS project, being guided by Dr. Hany Ammar as my research advisor. I would like to express my special thanks to him. His valuable comments and feedback were very valuable to me.

I express my heartfelt thanks to Mr Diaa, who has been guiding me right from the beginning of my research work. His continuous support, sound advices and valuable time were very vital to the quality of this research. I would not have reached this stage in research without his support. I would like to extend my vote of thanks to Dr. Li and Dr. Gamal Fahmy for serving on my committee.

I am very thankful to my parents, brother and sister for their blessings and continuous support throughout my period of study. Without their support I would not have reached so far.

I would also like to thank my husband for his active support and encouragement.

I am very thankful to all my friends and roommates for listening to my problems and extending their helping hand during the hour of need.

Once again my hearty thanks to all of you. 


\section{TABLE OF CONTENTS}

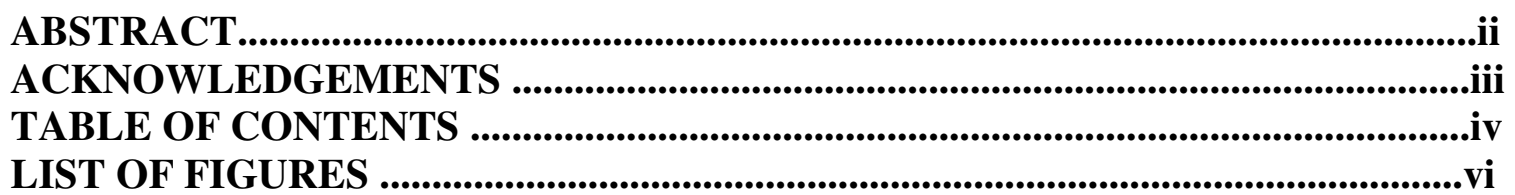

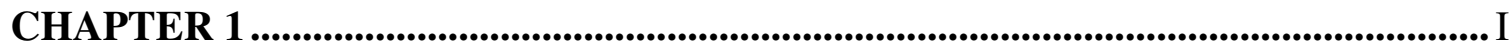

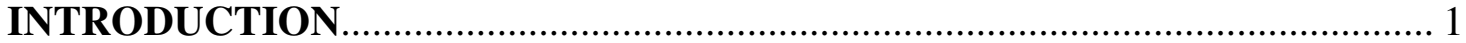

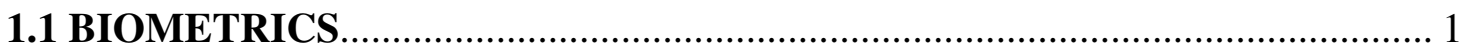

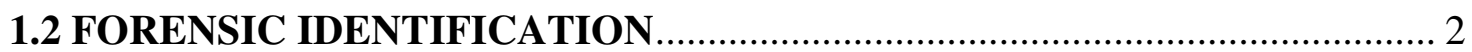

1.3 AUTOMATED DENTAL IDENTIFICATION SYSTEM .......................... 3

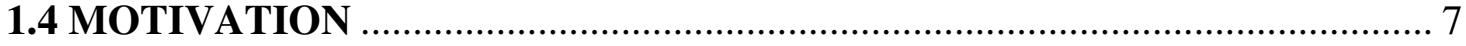

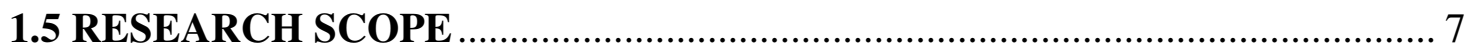

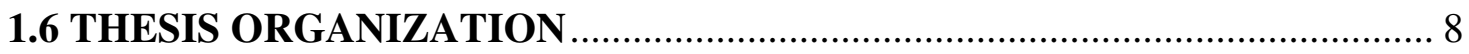

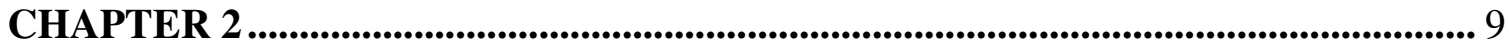

LITERATURE REVIEW AND RELATED WORK ................................................ 9

2.1 INTRODUCTION TO IMAGE REGISTRATION .................................... 9

2.2 APPLICATIONS OF IMAGE REGISTRATION ...................................... 10

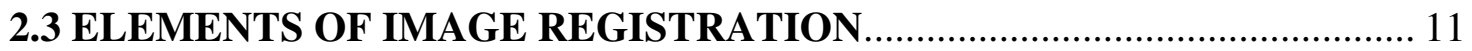

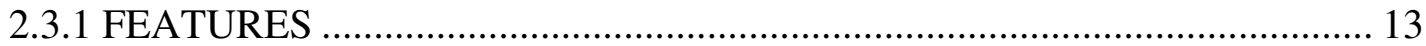

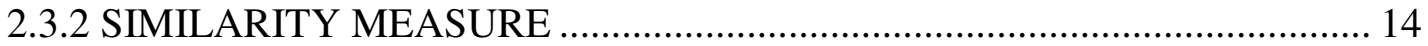

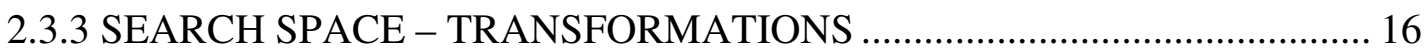

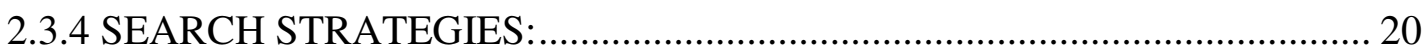

2.4 FEW IMAGE REGISTRATION METHODS ..................................... 23

2.5 PREVIOUS RESEARCH ON DENTAL IMAGE REGISTRATION ............ 25

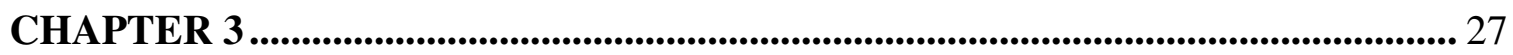

PROBLEM STATEMENT AND RESEARCH OBJECTIVES ................................. 27

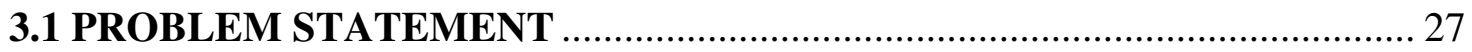

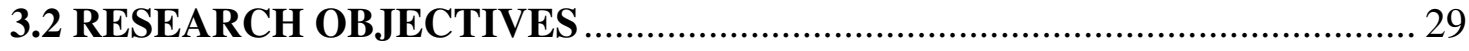

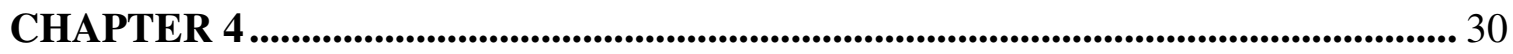

MULTI RESOLUTION DENTAL IMAGE REGISTRATION BASED ON

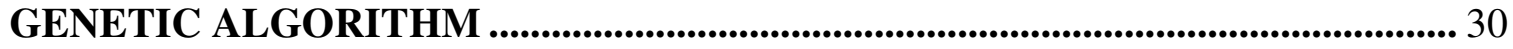

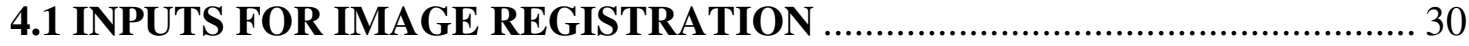

4.1.1 ELEMENTS OF DENTAL IMAGE REGISTRATION …........................... 31

4.2 EDGE FEATURE EXTRACTION …........................................................ 33

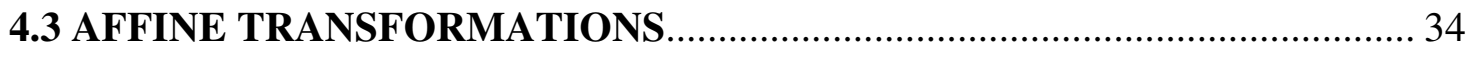

4.3.1 BOUNDS ON PARAMETERS OF AFFINE TRANSFORMATION ........... 36 


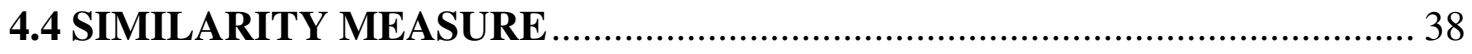

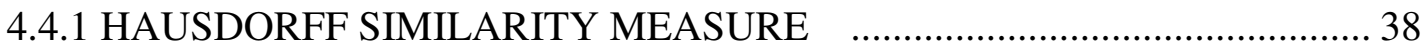

4.4.2 DISTANCE TRANSFORM MAP......................................................... 39

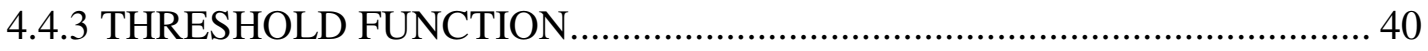

4.4.4 SIMILARITY MEASURE CALCULATION …........................................... 40

4.5 SEARCH STRATEGY - GENETIC ALGORITHM ………......................... 41

4.5.1 SEARCH USING GENETIC ALGORITHM ………................................... 42

4.5.2 COMPONENTS OF GENETIC SEARCH ……….................................. 42

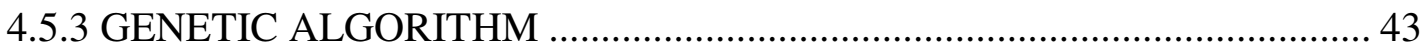

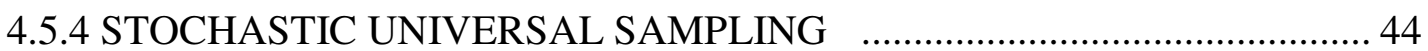

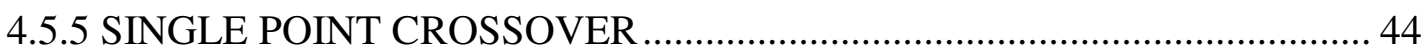

4.6 SEARCH DATA REDUCTION USING MULTI RESOLUTIONS ……........ 45

4.6.1 SUB -SAMPLING USING INTERPOLATION ............................................ 45

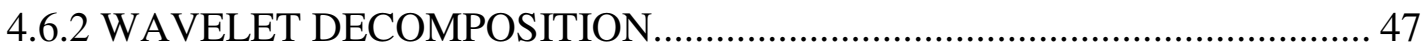

4.7 THE REGISTRATION TECHNIQUE....................................................... 50

4.7.1 REGISTRATION IN FINER LEVELS ................................................ 52

4.7.2 TERMINATION CRITERIA ……………………………………........ 54

4.8 ADVANTAGES OF MULTI-RESOLUTION GA SEARCH......................... 55

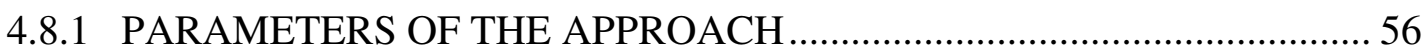

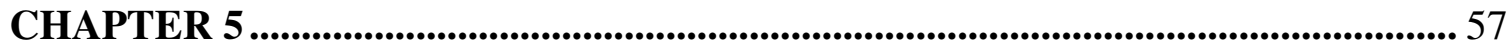

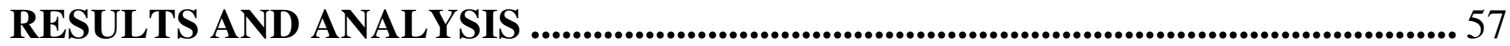

5.1 TESTING WITH UNKNOWN TRANSFORMATION PARAMETERS...... 58

5.2 TESTING WITH KNOWN TRANSFORMATION PARAMETERS ............ 60

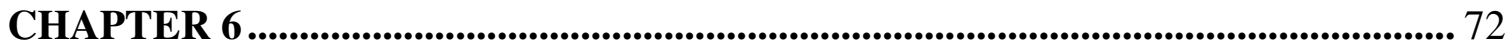

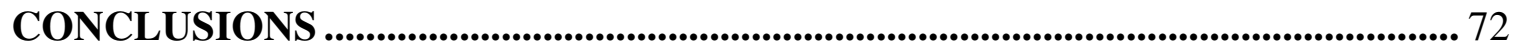

CHAPTER 7 ............................................................................................................................ 74

FUTURE WORK ................................................................................................................ 74

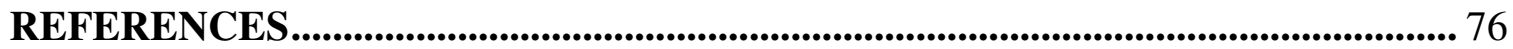




\section{LIST OF FIGURES}

Figure 1. 1 Block Diagram of ADIS ........................................................................................4

Figure 1. 2 Image Comparison Component ...............................................................6

Figure 2. 1 Elements of Image Registration ......................................................12

Figure 4. 1 Dental Region of Interest ......................................................................31

Figure 4. 2 Elements of Dental Image Registration ..............................................32

Figure 4. 3 Edge and Gradient Dental ROI............................................................34

Figure 4. 4 Affine Transformations......................................................................................36

Figure 4. 5 Distance transform map of Dental ROI .................................................39

Figure 4. 6 Similarity function of the algorithm ..........................................................40

Figure 4. 7 Methods of Subsampling....................................................................46

Figure 4. 8 Multi Resolution Dental ROI's using subsampling with nearest neighbor interpolation .....................................................................................................................46

Figure 4. 9 Levels of Wavelet Decomposition.........................................................48

Figure 4. 10 Multi Resolution Dental ROI's using wavelet Decomposition .. .........48

Figure 4. 11 Block Diagram of Dental Image Registration ....................................49

Figure 4. 12 Zero Padded Reference Image .........................................................50

Figure 4. 13 Graph of Similarity measure and generations......................................52

Figure 4. 14 Registration of Dental ROI's using subsampling .............................53

Figure 4. 15 Registration of Dental ROI's using wavelet decomposition ..............53

Figure 4. 16(a,b,c) Registration termination with a ten minute limit ....................54

Figure 5. 1 Block Diagram for testing using unknown transformations ................59 
Figure 5. 2 Plots of RMSE and SNR for Dental ROI's...............................................60

Figure 5. 3 Plots of RMSE and SNR for different termination criterion ..............61

Figure 5. 4 Block Diagram for testing using known transformations.......................63

Figure 5. 5 Plots of RMSE and SNR for Dental ROI's..................................................64

Figure 5. 6 Plots of RMSE and SNR for different termination criterion ..............65

Figure 5. 7 Plots of Average MSE and RMSE on transformation parameters ......66

Figure 5. 8 Plots of Average Relative Percentage errors on parameters..............67

Figure 5. 9 Example using Unknown Transformation Parameters .........................68

Figure 5. 10 Example using known Transformation Parameters ..............................69

Figure 5. 11Unregistered Pairs due to high variation between subject and reference........................................................................70

Figure 5. 12 Unregistered Pairs due to Partial Visibility of teeth and heavy misalignment.......................................................................71 


\section{CHAPTER 1}

\section{INTRODUCTION}

During the recent years various personal identification systems were invented and deployed to prevent fraud and aid criminal investigation and law enforcement authorities. We observe that research work in the area of biometrics is rapidly progressing primarily due to escalating security concerns worldwide. In sections 1.1 and 1.2 we give an overview of biometric identification systems. In section 1.3 we outline the research framework of the Automated Dental Identification System (ADIS), followed by motivation towards our research in section 1.4. We discuss the scope of our research in section 1.5 and section 1.6 consists of the thesis organization.

The two identification systems referred are Biometrics and Forensic Identification.

\subsection{BIOMETRICS}

Biometric science refers to the automatic identification of a person based on his/her physiological or behavioral characteristics [Jain99]. These identification characteristics include fingerprint verification, retinal and iris scanning, hand geometry, facial recognition, signature verification etc.

These identification techniques play a significant role in the areas where it is required to find the true identity of an individual. These techniques were employed primarily in high security applications such as financial services, health care, law enforcement, Government applications, travel and immigration, criminal identification, citizen identification, E-commerce and telephony. Physical presence of a person is required at the identification process. Advantage of this system is that there is no need to carry an identification tag or remember a password for identification [Jain99]. 


\subsection{FORENSIC IDENTIFICATION}

Forensic identification is another branch of science that uses natural sciences to help solve several legal cases and public issues. These include bank robberies, homicides and kidnapping cases [RM03]. In such situation the evidences found at the crime site such as blood, hair, fingerprints, bullets are considered for investigation process. Thus forensic identification comes into picture after some event has occurred.

Biometric identification is used before the occurrence of an event such as gaining access to any system, verification process etc, where as forensic identification is done after the occurrence of the event like finding historical information and analyzing criminal events to help jurisdiction in identifying criminals [RM03]. Forensic identification may take place prior to death and is referred to as Ante mortem (AM) identification. Identification carried out after death and is called Postmortem (PM) identification.

A postmortem biometric identifier has to survive severe conditions like air crash disasters, delays in discovery of dead bodies and resist early decay that affects body tissues. These forensic methods are used to identify the dead and missing people. Some of the forensic identification methods used are visual, finger/foot prints, DNA and dental systems.

In the case of visual assessment there may be a high possibility of affect of factors like emotional stress and constraints of members involved in the scene. Hence this is unreliable. In the case of finger / foot prints, hazards like fire, water etc can wipe away postmortem evidences. Obtaining fingerprints from victims and criminals also proved difficult and time consuming. DNA identification is being used in some cases but proves to be expensive and needs to tackle legal issues that arise.

Dealing with dental features for identification purpose is known as Forensic Odontology. Dental knowledge is applied in the field of civil and criminal investigations. Most forensic scientists consider dental features acceptable for postmortem identification. This 
is due to the fact that dental features are unique, i.e. no two persons can have same dental dentitions and sometimes identification may be possible with just a single tooth. Also teeth can be more durable and mostly remain unaffected by fire, decomposition or any medical trauma.

This research work is one such forensic identification system called Automated Dental Identification System where dental records are taken as basis for the identification of dead and missing people.

\subsection{AUTOMATED DENTAL IDENTIFICATION SYSTEM}

The research framework of Automated Dental Identification System (ADIS) aims at developing automated system for postmortem identification of individuals by comparing their Ante mortem (AM) and Postmortem (PM) dental records. The development of ADIS is based on digital image processing techniques such as image classification, image segmentation, feature extraction, image matching etc and research is being done to find accurate ways of applying them to dental radiographs.

Given a dental record of the subject the system aims at finding a list of candidates that possess dental features matching closely or identical to that of the subject. The forensic experts then decide which of the few candidates the subject is. The dental radiographs of a person taken from remains of death are used as Post mortem images. The PM/AM image is treated as subject image and thus a list of candidates are selected from dental records which have high similarities with that of the subject image. The Ante mortem images and Post mortem images are taken as the reference image and the input images. The input image is aligned with the images in the reference image database to find the best match.

Figure 1.1 shows the block diagram of the Automated Dental Identification System. 


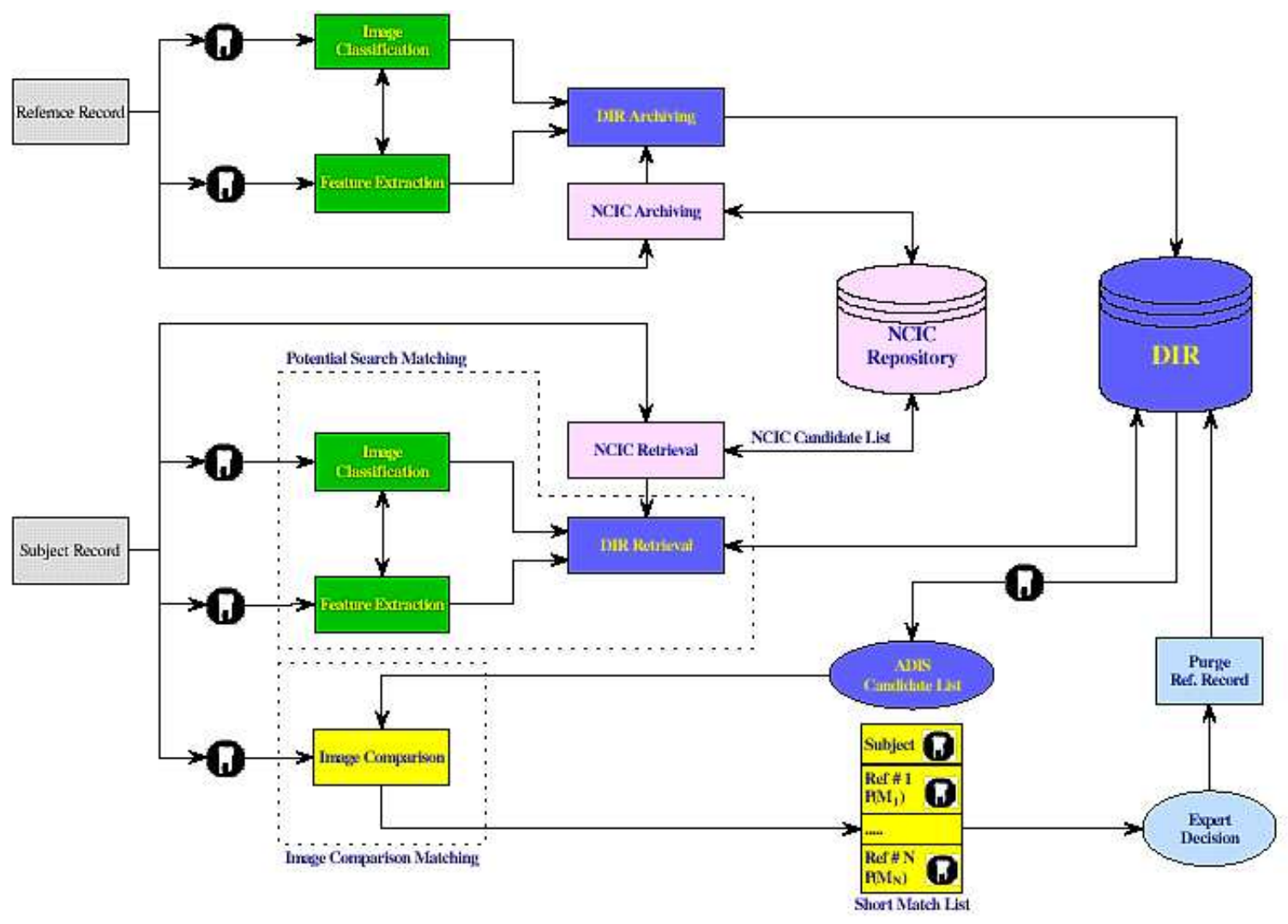

Figure 1.1 Block Diagram of ADIS

When a user submits a subject record that is typically an unidentified PM record, the NCIC codes are extracted from the subject image and high level features are extracted from the images. Both these features are used to extract a list of possible images from the DIR. DIR is seen in the upper portion of the figure. It is a collection of all image files and radiographs stored on the database server. The NCIC repository shown in the figure contains technical textual dental codes. The DIR and the NCIC repository are used during several stages like image comparison, updating repositories etc.

Both of them produce a list of candidate records that is passed to two main components in ADIS, the potential search matching component and the image comparison matching 
component. The potential search matching component in the lower portion of the figure is based on feature extraction and archival retrieval techniques, in which a set of features are extracted from the images, encoded as a feature template, and then used as a query for searching the dental feature database for the records with the most similar features and other attributes.

Once the list of candidate images are created, they are fed to the image comparison stage that selects a smaller list of candidate images for possible positive matches with the submitted subject image. This smaller list is then given to the forensic expert who makes the final decision for positive identification. Once a positive identification is made, for a subject image, then its corresponding reference image is removed from the DIR. If a positive identification cannot be made for a subject image, then this image is added to the DIR as a new missing or unidentified image. In this system, typically if the subject record is $\mathrm{AM}$, then the reference records are PM and vice versa.

\section{The Image Comparison Component [Nassar03]}

This component of ADIS system is being developed at West Virginia University. The subject images are compared with the reference images that are contained in the candidate list of ADIS. The outputs of comparison are ranked with their probability of match with the subject dental image. Comparing a pair of dental radiographs consists of two stages as follows:

a) Preprocessing the pair of radiographs

b) Decision-making

The image comparison component is shown in figure 1.2. 


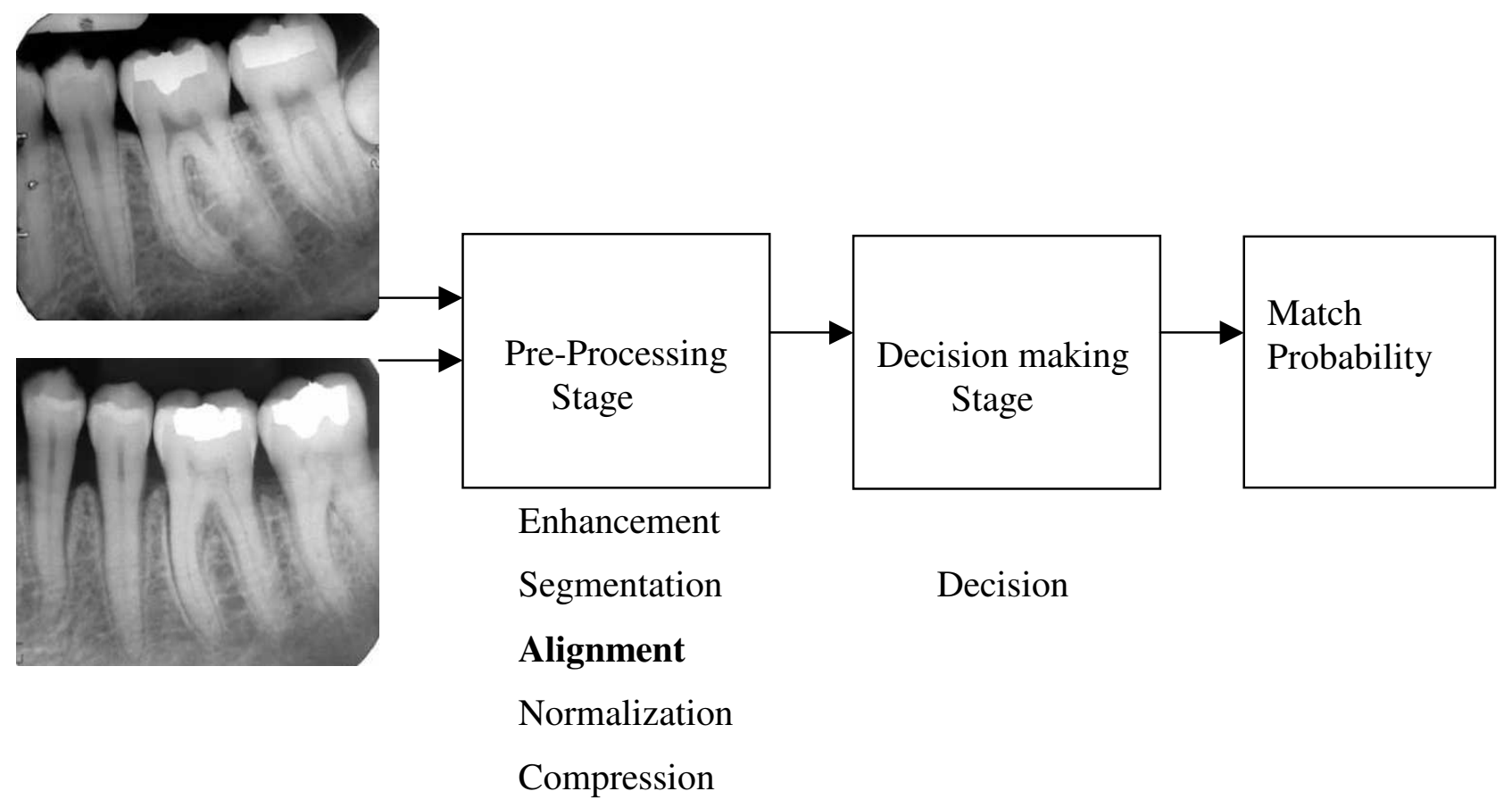

Figure 1.2 Image Comparison Component [Nassar03]

\section{Preprocessing}

The objective of Preprocessing stage is to out put a set of aligned images and compressed regions of interest(ROI) pairs with an input of two radiographic films (one from the subject record and the other from the reference record). These are then presented to the decision-making stage. Preprocessing stage consists of five layers namely Enhancement, Segmentation, Alignment, Normalization and Compression.

Some images in the DIR may have bad quality containing noise that make extracting teeth difficult. Enhancement reduces the noise in images and definition of teeth with respect to their surroundings is improved. After enhancement segmentation is done to separate the regions such that each region contains only single tooth. Hence in this step the regions of interest that possess highly distinctive features are extracted. Alignment step aligns the extracted subject regions of interest over their respective regions in the 
candidate image. Normalization is done on the scale and size of all regions. Compression removes some redundancy from the submitted pair to cut down on the computational complexity for pair matching in the decision making stage [Nassar03].

\section{Decision making}

The decision-making stage determines the probability of match between subject and reference films based on comparison of features extracted from both films.

\subsection{MOTIVATION}

In this thesis we are motivated to study the image registration step to develop a fast and robust algorithm for an efficient registration of the dental regions of interest. Throughout the remainder of this thesis we use the terms subject, query, and input images interchangeably to refer to a subject dental region of interest which is to be aligned with a reference dental region of interest. We emphasize on accuracy and speed to develop a competent approach to obtain desired registration results.

\subsection{RESEARCH SCOPE}

The work in this thesis is limited to alignment of 2D regions of interest extracted form digitized dental radiographs. The similarity score for image registration is based on edges and gradients of images. We consider translation, rotation, scale and shear factors during the transformation of input/subject image. Thus our transformation is limited to affine transformations. The Wavelet transform used for wavelet decomposition of images is also limited to some specified filters and interpolation is limited to nearest neighbor interpolation. The genetic algorithm used as the search strategy is limited to a simple basic algorithm. 


\subsection{THESIS ORGANIZATION}

The remaining chapters of the thesis are organized as follows. In chapter two we give a description about the previous research and related registration methods. Chapter three contains a brief description stating the problem and the research objectives. In chapter four we demonstrate our approach in a detailed manner consisting of inputs, theory of approach, bounds on parameters and the implemented technique. We also present diagrams and flow charts depicting various concepts. Chapter five presents testing methods and investigates the results. In chapter six and seven we discuss conclusions to our approach and predict the scope of future work. 


\section{CHAPTER 2}

\section{LITERATURE REVIEW AND RELATED WORK}

Owing to its wide range of applications, numerous techniques for image registration are being developed and classified based on their elements such as feature space, similarity measures and search strategies. In this chapter we present an introduction to theory of image registration in section 2.1. In section 2.2 we elucidate the applications of image registration in various fields. Section 2.3 contains a detailed description of its elements with a wide range of research works along with their advantages and disadvantages. In section 2.4 we review few image registration techniques followed by previous work in dental image registration in section 2.5.

\subsection{INTRODUCTION TO IMAGE REGISTRATION}

Image Registration plays a key role in image processing for matching of images that differ over a certain period of time or that have been distorted due to various reasons. Alignment technique should determine the changes in scenes of objects taken under different conditions and times and accordingly restore misalignment. Thus to align the images a transformation should be found to map each point in one image to a point in the other [Brown92].

If two images that need to be aligned are defined as two dimensional arrays of some size denoted by $I_{1}$ and $I_{2}$ where $I_{1}(x, y)$ and $I_{2}(x, y)$ map to intensity values, then the mapping between the images can be expressed as:

$$
\mathrm{I}_{2}(\mathrm{x}, \mathrm{y})=\mathrm{g}\left(\mathrm{I}_{1}(\mathrm{f}(\mathrm{x}, \mathrm{y}))\right) \quad \text { Eqn }(2.1)
$$


Where $\mathrm{f}$ is a $2 \mathrm{D}$ spatial coordinate transformation and $\mathrm{g}$ is $1 \mathrm{D}$ intensity transformation. [Brown92]

Image registration techniques are classified based on the feature matching method, similarity measures and search strategies used. Successful image registration requires a thorough knowledge about the causes and sources of misalignment.

\subsection{APPLICATIONS OF IMAGE REGISTRATION}

Here we present some fields where image registration is being utilized.

Biometric \& Forensic systems: As discussed in the earlier chapter, image registration plays a significant role in providing successful solutions to the field of certain biometric systems for tracking of characteristics of individuals and locating patterns such as finger printing, retinal features, signature verification, character recognition and facial recognition [Brown92].

Remote Sensing: Locating a pattern or model in an image by image registration finds application in remotely sensed data processing that consists of locating positions and orientations of features such as runways, terminals and parking lots. Mosaicing images of the surveyed area and registration of aerial or satellite data into maps and other GIS layers is done using image registration for remote sensing. [Brown92][Zitova03]

Computer vision: Registration of images taken from different viewpoints finds applications in depth and shape reconstructions for stereo mapping in the field of computer vision and tracking object motion. Automatic detection of change for security monitoring, target template matching with real time images and quality inspection are the other applications of image registration in the field of computer vision. [Brown92] [Zitova03] 
Medical Image Registration [Kneöaurek00]

Image registration plays a vital role in the field of medicine. Some areas of medicine where image registration is used are discussed below.

a) Medical diagnosis requires usage of SPECT and PET. Uncertainty in the anatomic definition on SPECT and PET images, however, sometimes limits their usefulness. To overcome this problem, a combination of magnetic resonance images (MRI) and X-ray computed tomography (CT) images with functional SPECT or PET images of the same sections of the body is used. This provides complementary anatomic (MRI or CT) and physiological (SPECT or PET) information useful for research, diagnosis, and treatment [Kneöaurek00].

b) Early detection of cancers is another major application of registration. It also works as a visualization tool that can significantly aid in the early detection of tumors and other diseases, and aid in improving the accuracy of diagnosis.

c) Diagnosis of breast cancer, colon cancer, cardiac studies, wrist and other injuries, inflammatory diseases, different neurological disorders including brain tumors, Alzheimer's disease and schizophrenia, radiotherapy for brain tumors etc are dealt using image registration. [Kneöaurek00].

d) Digital subtraction angiography where in registration of images is done before and after radioisotope injections, detection of cataract and tumors.

Other applications: Other applications include integrating images from various electromagnetic bands like in microwave, radar, scene classification such as classifying buildings, roads, vehicles and surveillance of nuclear plants.

\subsection{ELEMENTS OF IMAGE REGISTRATION}

Any image registration method is a combination of the following four steps. All registration techniques can be viewed as different combinations of these steps. They are:
a) Features
b) Similarity measure
c) Search Space \& Transformations 
d) Search Strategy

The figure 2.1 below depicts the elements of image registration.

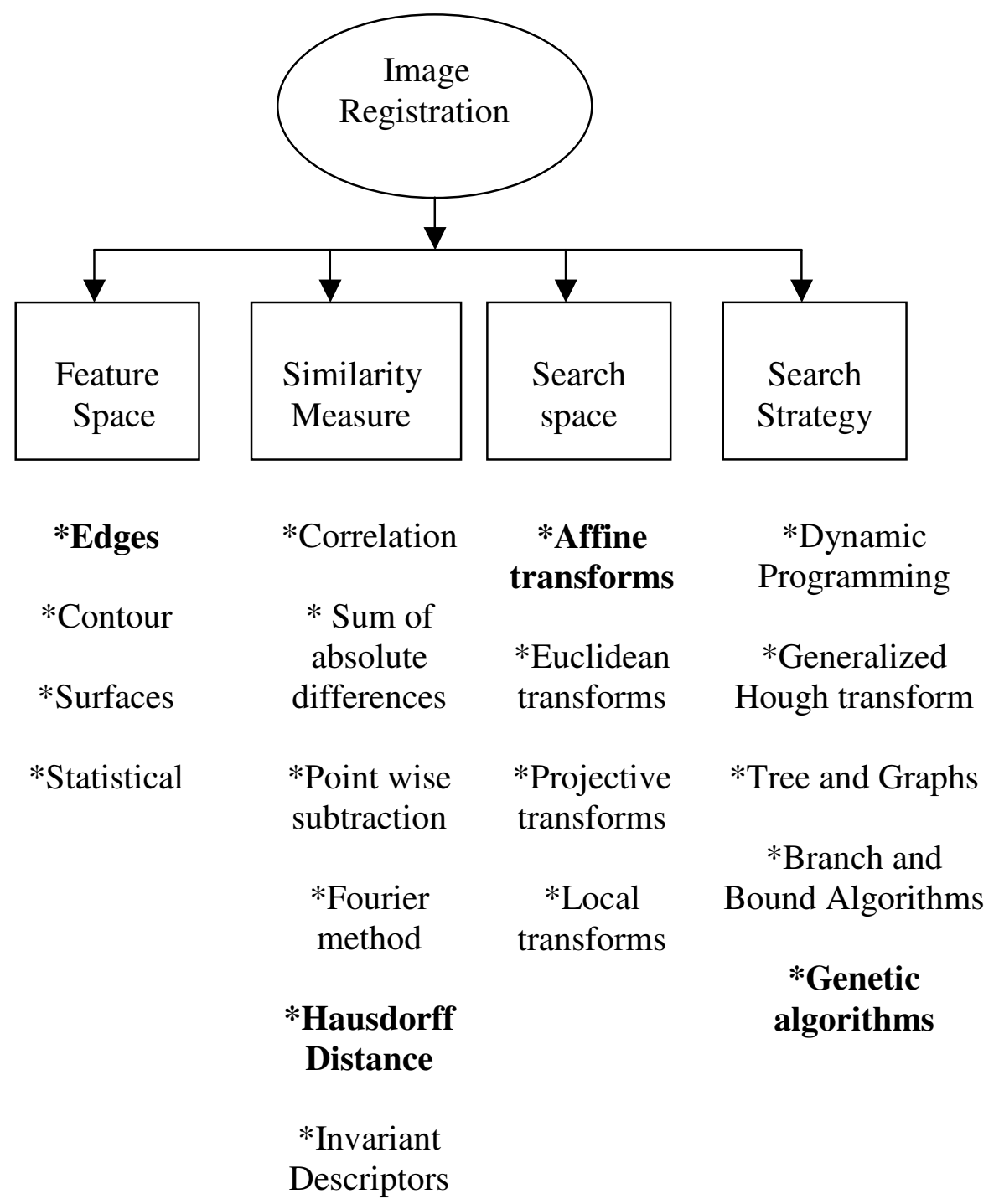

Figure 2.1 Elements of Image registration 


\subsubsection{FEATURES}

Features are the data used for registration. They are computed prior to matching on both images. Among these edges and contours represent most of the intrinsic structure of the image, less sensitive to noise, and spread over the image, hence are most frequently used [Brown92]. Statistical features like moment invariants and centroids use all of the information for registration. Some salient features include Fourier descriptors, centers of gravity of closed boundary regions, line intersections. Graphs of patterns and grammars composed of patterns are the some type of features used [Zitova03]. Some medical imaging methods use line representations as features. Canny edge detectors, Laplacian of Gaussian, Sobel edge operator etc are commonly used edge detection methods. Point feature methods consider line intersections as points that represent features [Zitova03]. The features detected in image influence the computational cost of image registration process by reducing the search space and by increasing only relevant computations.

If the features in the images to be detected are unclear or distorted then registration methods using spatial relations can be used. Goshtasby computed features in the subject images, which are near the range of the features of the reference image and transformation parameters with more score are taken as good estimates. [Goshtasby85]

Stockman in [Stockman82] presented a technique called the Clustering technique. The feature points in this method are the points that connect edges. Selecting the control points, the transformation is found which maps the points of the reference and the subject image.

Chamfer matching [Barrow77] proposed by Barrow is an edge matching technique in which the edge points of one image are transformed to edge points of similar image that has been distorted. The best fit for the edge points is then found out by minimizing a generalized distance between them. 


\subsubsection{SIMILARITY MEASURE}

The choice of the similarity metric plays a vital role in determining the registration transformation. This step performs the task of finding the relative match between the reference and subject images. Similarity measure selection should take into account the factors like false feature detection, degradation of images, dissimilarity of images due to different imaging conditions. Here we discuss some similarity measures used.
a) Correlation method
b) Sequential similarity detection algorithm
c) Fourier methods
d) Point wise subtraction of images
e) Hausdorff Distance
f) Invariant Descriptors

Correlation method: It is a match measure that gives the degree of similarity between image and a template. The value of cross correlation over all possible translations gives the measure of similarity for window pairs. [Zitova03]

Sequential Similarity detection algorithm: Barnea and Silverman [Barnea72] developed this algorithm that accumulates sum of absolute differences of the image intensity values and applies a threshold criterion, if the accumulated sum exceeds a given threshold; the candidate pair of windows from both images is rejected [Zitova03]. This decreases the complexity of computation with less degradation of performance. It maintains reasonable time complexity and efficiency is improved compared to the correlation methods.

Fourier methods: These methods work for images obtained under varying conditions and containing noise. The phase correlation method aligns images, which are shifted relative to one another [kuglin75]. It is based on Fourier shift Theorem. This method proves successful for images from different sensors and images obtained under different 
illumination conditions. This method reduces time to compute the match measure for large images.

DeCastro and Morandi introduced an extended phase correlation to incorporate rotational transform. Images with scale changes were registered using polar log mapping of spectral magnitude and phase correlation [Castro87]. This combination helped registration of affinely distorted images.

Point wise subtraction of images: In a similarity measure suggested by Venot in [Venot 84] the two images that are required to be aligned are subtracted from one another and if the number of sign changes is high, the images are considered to be aligned. It assumes that a point has equal probability of being above or below zero.

Distance similarity measure: The Hausdorff distance measures the extent to which each point of a model set lies near some point of an image set and vice versa. Thus, this distance can be used to determine the degree of resemblance between two objects that are superimposed on one another. The method is quite tolerant of small position errors such as those that occur with edge detectors and other feature extraction methods. [Huttenlocher93]

The measure of similarity between the can be viewed as a multidimensional optimization method where the degrees of freedom measure of the transformation represent the number of dimensions.

Invariant descriptors: The correspondence between features in the images can be described using their description. Invariant descriptions are termed as ones that describe the same corresponding features in both the reference and subject images. Usually the 
minimum distance rule with threshold is applied while searching for the best matched feature pairs in the space of feature descriptors.[Zitova03]

In the work by Chellappa and Zheng [Zheng93] the estimation of illuminant direction is first used to obtain an estimate of initial camera rotation. The scale and translation parameter estimates are done using pair wise matching of feature points. Finally correlation based matching is done to obtain an accurate estimation of final registration parameters.

Moment based invariants to the similarity transform were introduced and used for registration. Sato and Cipolla used circular moments of distribution of the line features orientation to compute parameters of geometric deformations. [Sato95]

Chandrasekhar, Govindu and Chellappa addressed the problem of registering images obtained from different views and lighting conditions. In their method first the transformation is divided into sequence of stages and at each stage appropriate feature class is selected and the value of transformation parameter is estimated that is consistent with it. The value of parameter that is consistent with most feature pairs is considered to be the best estimate. This method is robust to variations in sensor characteristics and imaging conditions. Invariant descriptors can be used in situations where no prior feature detection is done. [Shekhar99]

\subsubsection{SEARCH SPACE - TRANSFORMATIONS}

The search space is the space of all possible transformations of an image. The characteristics of search space are determined by the transformations used. Selection of transformation for a particular alignment problem depends on the basic cause of misalignment between the two images [Brown92]. A transformation is needed to map one image onto the other. The transformation class used for the registration should be 
able to eliminate the geometric distortions present in the image. Here we give a brief description of the basic operations in geometric transformations.

Translation: It is the vertical or horizontal shift of an image coordinate points to another set of points.

Rotation: In this transformation the points of an image are rotated by an angle ' $\theta$ '.

Scaling: Scaling transformations stretch or shrink a given coordinate system and as a result change lengths and angles.

Shearing: Shearing produces a relative horizontal or vertical displacement, resulting in a sort of leaning effect.

Transformations are classified into the following:
a) Global Transformations
b) Local Transformations

\section{Global Transformations}

For a global transformation only one equation is used that maps the entire image [Brown92]. Examples for this type are the affine, projective, polynomial, Euclidean transformations. Affine and Euclidean are the most commonly used transformations. We give a brief description of some common global transformation techniques used.
a) Euclidean Transformations
b) Affine Transformations
c) Projective Transformations

Euclidean transformations: Euclidean transformations are composed of translation and rotation parameters. They preserve length and angle measure. The shape of an object will not change. That is, lines transform to lines, planes transform to planes, circles transform 
to circles, and ellipsoids transform to ellipsoids. Only position and orientation of the object will change. Considering $\left(\mathrm{x}_{1}, \mathrm{y}_{1}\right)$ as coordinate points of an image and $\left(\mathrm{x}_{2}, \mathrm{y}_{2}\right)$ as points of the transformed image, the equation of Euclidean transformation in matrix form is:

$$
\left(\begin{array}{l}
x_{2} \\
y_{2}
\end{array}\right)=\left(\begin{array}{cc}
\cos \theta & \sin \theta \\
-\sin \theta & \cos \theta
\end{array}\right)\left(\begin{array}{l}
x_{1} \\
y_{1}
\end{array}\right)+\left(\begin{array}{l}
a \\
b
\end{array}\right)
$$

[Millwala04]

Eqn (2.2)

Here ' $\theta$ ' specifies the angle of rotation where ' $a$ ' and ' $b$ ' are translation parameters.

Affine transformations: In $2-\mathrm{D}$ geometric transformations an affine transformation maps variables in input image to new variables in output image by applying a linear combination of translation, rotation, scaling, and/or shearing. Hence it is equivalent to the composed effects of translation, rotation, scaling and shear.

This transformation maps parallel lines to parallel lines, but circles become ellipses. It does not necessarily preserve angles or lengths [Jain86]. Considering $\left(\mathrm{x}_{1}, \mathrm{y}_{1}\right)$ as coordinate points of an image and $\left(\mathrm{x}_{2}, \mathrm{y}_{2}\right)$ as points of the transformed image, the equation of an affine transformation in the matrix form is:

$$
\left(\begin{array}{l}
x_{2} \\
y_{2} \\
1
\end{array}\right)=\left(\begin{array}{ccc}
a & b & e \\
c & d & f \\
0 & 0 & 1
\end{array}\right)\left(\begin{array}{l}
x_{1} \\
y_{1} \\
1
\end{array}\right)
$$

The six constants ' $\mathrm{a}$ ' through 'f' determine the effect of transformation. 
Projective transformations: A transformation that maps lines to lines, but does not necessarily preserve parallelism. Considering $\left(\mathrm{x}_{1}, \mathrm{y}_{1}\right)$ as coordinate points of an image and $\left(x_{2}, y_{2}\right)$ as points of the transformed image, the equation of a projective transformation in the matrix form is:

$$
\left(\begin{array}{l}
x_{2} \\
y_{2} \\
z_{2}
\end{array}\right)=\left(\begin{array}{lll}
a & b & c \\
d & e & f \\
g & h & i
\end{array}\right)\left(\begin{array}{l}
x_{1} \\
y_{1} \\
z_{1}
\end{array}\right)
$$

Eqn (2.4)

Thus projective transformations contain nine independent parameters.

\section{Local Transformations}

Local transformations map the image depending on their spatial location [Brown 92]. The global mapping models described above do not properly transform images that contain local distortions. Local mapping models are more powerful when compared to global ones and can handle local distortions [Brown 92]. For these mapping models, many computations are done, either for each local piece or spreading them to neighborhoods. Local mapping methods can be used in cases that include threedimensional scenes taken from different viewpoints, and effects of different sensor conditions or scene conditions.

a) Piece wise interpolation: This technique is used for finding local distortions by point matching in which a spatial transformation is specified for each coordinate that interpolates between the matched coordinate values. A general spline approach is used for this that requires selection of a set of basic functions and a set of 
constraints that need to be satisfied so that solving a system would specify the interpolating function. [Brown92].

b) Weighted Sum: For a simpler case weighted sum of neighboring points is calculated where the weights are inversely related with distance. Local weighted mean depends on the number of control points, order of polynomial and size of the image. The method proved good efficiency but selection of control points plays a major role in determining its success. [Brown 92]

\section{Radial based mapping}

These mapping functions come under the group of global mapping methods, but handle geometric distortions that vary locally [Zitova03]. Some examples of functions used in this mapping are multiquadrics, Gaussians, thin-plate splines etc. Thin plate splines are commonly used radial basis functions. A thin plate spline can be considered as a very thin plate which is fixed at positions that are determined by control points in the reference image in the heights given by $\mathrm{x}$ or $\mathrm{y}$ coordinates of corresponding control points in the subject image. A thin plate spline minimizes the quadratic variation functional of potential energy that reflects the amount of function variation and should be small for good mapping function [Zitova03].

\subsubsection{SEARCH STRATEGIES:}

An efficient search strategy is required to search for best transformation that gives maximum similarity metric between the subject and reference images. Reducing the computational cost plays a vital role in determining the efficiency of registration method used. Therefore, a suitable search strategy must be designed to decrease the number of features that need to be matched and reduce search space. Some of the search strategies are as follows:
a) Dynamic Programming
b) Generalized Hough Transform 

c) Tree and Graph Matching
d) Search strategy of Branch and Bound Algorithms
e) Genetic Algorithms

Dynamic Programming: This is the most commonly used algorithm search strategy for registration. The solutions to sub problems are used effectively in order to solve the larger ones. This has been applied for signature verification, stereo mapping and shape matching. It is a search for the lower cost path that matches each point along the contour of one image along that of another [Brown 92]. Dynamic programming reduces the resultant search space and cost by limiting the matches to an interval size, which is the largest expected difference between the images. This search strategy matches images that contain noise effectively.

Generalized Hough Transform: The Generalized Hough transform based approach to point pattern matching converts point pattern matching to a problem of detecting peaks in the Hough space of transformation parameters. It discretizes the parameter space and accumulates the evidence in the discretized space by deriving transformation parameters that relate two point patterns using a feature matching technique. For all the derived transformations, matching score is computed. The transformation with the maximum score is taken as the correct one. Generalized Hough transforms are proved to be robust against occlusions, noise, distortions, local illumination changes and its capacity to handle multiple occurrences of shapes being detected. [Ballard81]

Tree and Graph matching: These matching methods use graph and tree properties to minimize the search. In the work by Bing Ma, Alfred Hero a graph method with Renyi entropy as the dissimilarity measure between images is proposed. Minimizing the length of a minimum spanning tree that spans the graph obtained from superimposed images does matching. If two images are properly aligned the Renyi entropy of over lapped images achieves its minimum and hence total length of the graph is shortest among all possible overlapping of images. This algorithm proved to be robust for noise contamination and minor differences in the images. [Hero00] 
Search strategies of Branch and Bound Algorithms: The Branch and bound algorithms work by subdividing the transformation space and placing bounds on the quality of match over each subdivision. In the work by Thomas M. Breuel two approaches are discussed to computing the bounds, the match list approach and the approaches based on point location data structures. A depth first Priority queue based search strategy is compared to the conventional best-first search strategy conventionally applied in the branch and bound algorithms. It was found out that the depth-first search strategy uses much less space than a best fit strategy. The depth first strategy gives a considerable reduction in the memory usage. The memory reduction was shown to be 50200 folds, with only a small growth in the running time. [Bruel02]

Genetic Algorithms: Genetic Algorithms are proved to be robust for search and optimization problems. They were invented by John Holland and developed by him and his students and colleagues. In the process of using GA, the process of finding solutions generates other points (possible solutions) as evolution proceeds.

Fitzpatrick [Fitz84] used the Genetic algorithmic search in determining the transformation of moving object between the images. Das Gupta and Mc Gregor proposed the structured genetic algorithm for the registration process. The structured genetic algorithm was claimed to be faster than the previous works of simple genetic algorithm [Chalermwat99]. Ammar used genetic algorithm to register finger prints and compared them with other algorithms. They proved that genetic algorithm was more faster and effective for finger print registration.[Ammar00] 


\subsection{FEW IMAGE REGISTRATION METHODS}

\section{Mutual Information methods}

These methods are used in the alignment of multimodal images and prove more applicable in the field of medical imaging. The method originates from information theory and is a statistical dependency between two data sets [Zitova03].

In the work by P.Thevenaz, M.Unser mutual information between two images was used for registration. They have proposed computing joint histogram required for mutual information using Parzen windows. They satisfy the partition of unity condition. Thus they could develop an optimizer based on Marqardt -Levenberg strategy. Validation of their work was done on brain images and comparing them with the previous works showed better accuracy due to consistency of the approach. [Thevenaz98]

In the work by Ritter et al pyramid sampling based re-annealing search was used to find the maximum of mutual information between images. This technique improved the speed and precision of registration. The algorithm pre determines precision of the process within limits that are imposed by noise levels of the data. This is subjected to choice of interpolation and time constraint. The algorithm showed success on 49 pairs of stereo images and 48 pairs of temporal images. [Ritter99]

Anand Rangarajan worked on rigid point registration using mutual information method. Their work focuses on the points of area borders as features. The main concept of their method is the emergence of correspondence between sets as a result of joint density estimation. They applied the algorithm to alignment of primate auto radiographs successfully. [Rangarajan99] 


\section{Wavelets and Pyramids}

The Pyramidal approach to image registration was introduced to reduce computations for large size image. The approach is a coarse to fine hierarchical strategy in which the registration method is applied initially on a coarse resolution and taking these estimates into consideration assumptions are made for the parameters of finer resolutions.

Pyramidal approach combined with wavelet decomposition proved to give good results. The wavelet decomposition decomposes the image into coefficient sets (LL, HL, LH, $\mathrm{HH}$ ) using the image filtering, i.e. using the low pass filter and high pass filter on the image.

Prachya Chalermwat used wavelet decomposition of images to extract LL, LH, and HL images and applied Cross Correlation to register images. They used Genetic Algorithm as the search strategy. Wavelet decomposition of images together with an efficient search strategy further speeded up the process of computations. This technique has been used to register Land Sat images. [Chalermwat99]

\section{Elastic Registration}

In these methods of registration the image is considered to be an elastic body and similarity among the features act as forces that stretch the body. These are counterbalanced by stiffness and smoothness constraints with minimal amount of bend and stretches. The features are matched and the transformation is determined. Registration is achieved by finding out the minimum Energy State in iterative manner. This method is used to register the images with enough complex and local distortions.

Bajcsy developed this idea. In their work [Bajcsy89] a set of partial differential equations are used as constraints. The external forces are computed as a gradient vector of similarity function. These external forces act on elastic body pulling it towards maximum similarity function. 


\section{Fluid Registration}

Fluid registration handles the issue of localized deformations in the image. The reference image is considered to be a thick fluid that flows out to match the subject image under the control of a derivative of the Gaussian sensor model Thus the viscous fluid model controls the image transformation. This method is mostly used for medical applications. The disadvantage of this method is the occurrence of blurring during registration process. [Zitova03].

\subsection{PREVIOUS RESEARCH ON DENTAL IMAGE REGISTRATION}

In this research we focus mainly on registration of dental regions of interest, under the image comparison component of the research framework of Automated Dental Identification System (ADIS). Few other researchers have performed dental image registration during the past few years. Here we present an outline of methods developed by them.

\section{Projective Geometry}

In the work by Ostuni $\mathbf{J}$ [Ostuni93] projective geometry was utilized to describe the position of an image pixel. A coordinate system called reference triangle was used to describe the pixels. It was proven experimentally that the method successfully registers the entire image with better performance in speed compared to the algorithm that used synthetic projective geometry.

\section{Digital Subtraction radiography}

The aim of D. C Yoon [Yoon00] was to provide a robust method which eliminates manual selection of reference points for registration. Edges were selected as the features for matching. They adopted a nearest neighboring criterion to pair up edge pixels of both dental radiographs. An affine transformation was used for aligning them and the transformation was determined that showed minimum spatial alignment error. The final transformation is then applied to the entire first image resulting in a close spatial match to 
the second image. They obtained a root mean square error that was $14 \%$ lower than the manual alignment technique.

\section{Fuzzy Strategy}

Fuzzy strategy for registration of dental radiograms was proposed in [Samad04]. They adopted parameters like geometric constraints, texture differences and utilized them for the decision making process, reducing the probability of mismatches. Fuzzy reasoning was employed in the stage of feature identification and modeling. Multi resolution representation of information was adopted. They obtained a set of transformations to register the reference and subject dental radiograms. This strategy proved to be efficient and reliable for dental radiogram registration. [Samad04] 


\section{CHAPTER 3}

\section{PROBLEM STATEMENT AND RESEARCH OBJECTIVES}

In this chapter we focus on stating the problem in section 3.1 followed by our research objectives in section 3.2 .

\subsection{PROBLEM STATEMENT}

Image Alignment is the process of overlaying two images of the same scene taken at different times, from different sensors or over different illumination conditions [Zitova03]. The aim of registration is to geometrically align two images, and correct misalignments with respect to geometric transformations. The final information of the registration process is a set of transformation parameters that map points of one image onto the corresponding points of the other image.

The Automated Dental Identification System (ADIS) uses dental features for postmortem identification. The research frame work Automated Dental Identification System (ADIS) requires an efficient image registration technique under the preprocessing layer of image comparison component for matching the AM and PM dental regions of interest.

Given a pair of input Anti mortem and Post mortem regions of interest, this research work aims at considering one of them as subject, the other as a reference and developing algorithms for aligning the subject and reference images. The registration step transforms the input region of interest to the corresponding reference region of interest in dental images.

Hence we require developing a fast and robust algorithm to deal with the noisy and distorted dental images. The developed algorithm should provide reasonable performance 
in terms of accuracy in results and speedy generation of transformation parameters. The error on the transformation parameters must be within specified limits.

The image registration problem can be formulated as:

\section{GIVEN}

Two dental regions of interest $\mathrm{P}$ and $\mathrm{Q}$, where $\mathrm{P}$ is the reference $\mathrm{ROI}$ and $\mathrm{Q}$ is the subject/input ROI. We need to transform $Q$ in such a way as to obtain a maximum similarity score between the reference dental region of interest i.e. $\mathrm{P}$ and transformed version of input dental region of interest $\mathrm{Q}$. The transformed version of $\mathrm{Q}$ can be termed as $\operatorname{Tr}(\mathrm{Q})$. The transformation with the maximum similarity measure would be the best transformation.

$\mathrm{x}_{\mathrm{Q}}$ and $\mathrm{y}_{\mathrm{Q}}$ be the coordinate points of region of interest $\mathrm{Q}$

$\mathrm{X}_{\mathrm{TrQ}}$ and $\mathrm{Y}_{\mathrm{TrQ}}$ be the coordinate points of the transformed version of $\mathrm{Q}$ i.e. $\operatorname{Tr}(\mathrm{Q})$.

Assuming the affine transformation model for transforming the query dental region of interest, the transformation equation can be written as:

$$
\left(\begin{array}{c}
X_{\operatorname{Tr} Q} \\
Y_{\operatorname{Tr} Q} \\
1
\end{array}\right)=\left(\begin{array}{ccc}
\mathrm{a} & \mathrm{b} & \mathrm{e} \\
\mathrm{c} & \mathrm{d} & \mathrm{f} \\
0 & 0 & 1
\end{array}\right)\left(\begin{array}{c}
\mathrm{x}_{\mathrm{Q}} \\
\mathrm{y}_{\mathrm{Q}} \\
1
\end{array}\right)
$$

\section{PROBLEM}

To estimate the transformation T satisfying the following condition:

$\mathrm{T}=\arg \min \mathrm{d}(\mathrm{P}, \operatorname{Tr}(\mathrm{Q}))$; 
$\mathrm{T}=\arg \max \mathrm{S}(\mathrm{P}, \operatorname{Tr}(\mathrm{Q}))$

(T)

Where $\mathrm{d}=$ distance, and $\mathrm{S}=$ similarity measure

\subsection{RESEARCH OBJECTIVES}

The objectives of this research are summarized as follows:

1) Develop techniques for registration of extracted subject regions of interest with corresponding reference regions of interest with a percentage relative error on the resultant parameters below $20 \%$.

2) The algorithms should be robust to register noisy dental regions of interest emphasizing on accuracy and speed of registration. 


\section{CHAPTER 4}

\section{MULTI RESOLUTION DENTAL IMAGE REGISTRATION BASED ON GENETIC ALGORITHM}

Digital image registration has become a topic of much recent research due to its wide range of applications. As discussed in earlier chapter image registration methods contain features, similarity metric, transformations and search strategy as their elements. In this chapter we describe the theory of elements adopted for our registration technique followed by our multi resolution approach for search data reduction.

In section 4.1 defines the inputs to our technique. Section 4.2 presents the feature extraction. Transformations adopted followed by bounds on the transformation parameters are elucidated in section 4.3. In section 4.4 we discuss the similarity measure used followed by search strategy in section 4.5. Section 4.6 consists of multi resolution approach used for search data reduction followed by section 4.7 that describes the registration technique. In section 4.8 we focus on the advantages and limitations of the approach developed.

\subsection{INPUTS FOR IMAGE REGISTRATION}

The Ante mortem (AM) and Post mortem (PM) dental regions of interest from the segmentation layer of the ADIS system are used interchangeably as the reference and query images. These gray scale dental images contain many perturbations, distortions and noise.

The dental region of interest used is shown in figure 4.1 below 


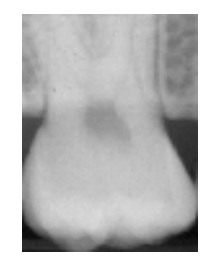

Figure 4.1 Dental region of interest

\subsubsection{ELEMENTS OF DENTAL IMAGE REGISTRATION}

We have adopted a multi resolution based image registration to register Dental regions of interest. Performing image registration on multi resolution versions of the subject and reference image was introduced to reduce computations for large size images, making the registration faster. Edges and Edge gradients are selected as features that need to be matched. We selected Oriented Hausdorff Similarity measure in our approach. This method utilizes edges, gradients of images, distance transforms to determine similarity between two images. We selected affine transformations to capture complex distortions. Genetic algorithm which proved as efficient search space reduction technique is used as a search strategy.

We present the elements adopted for our dental image registration in the figure 4.2. 


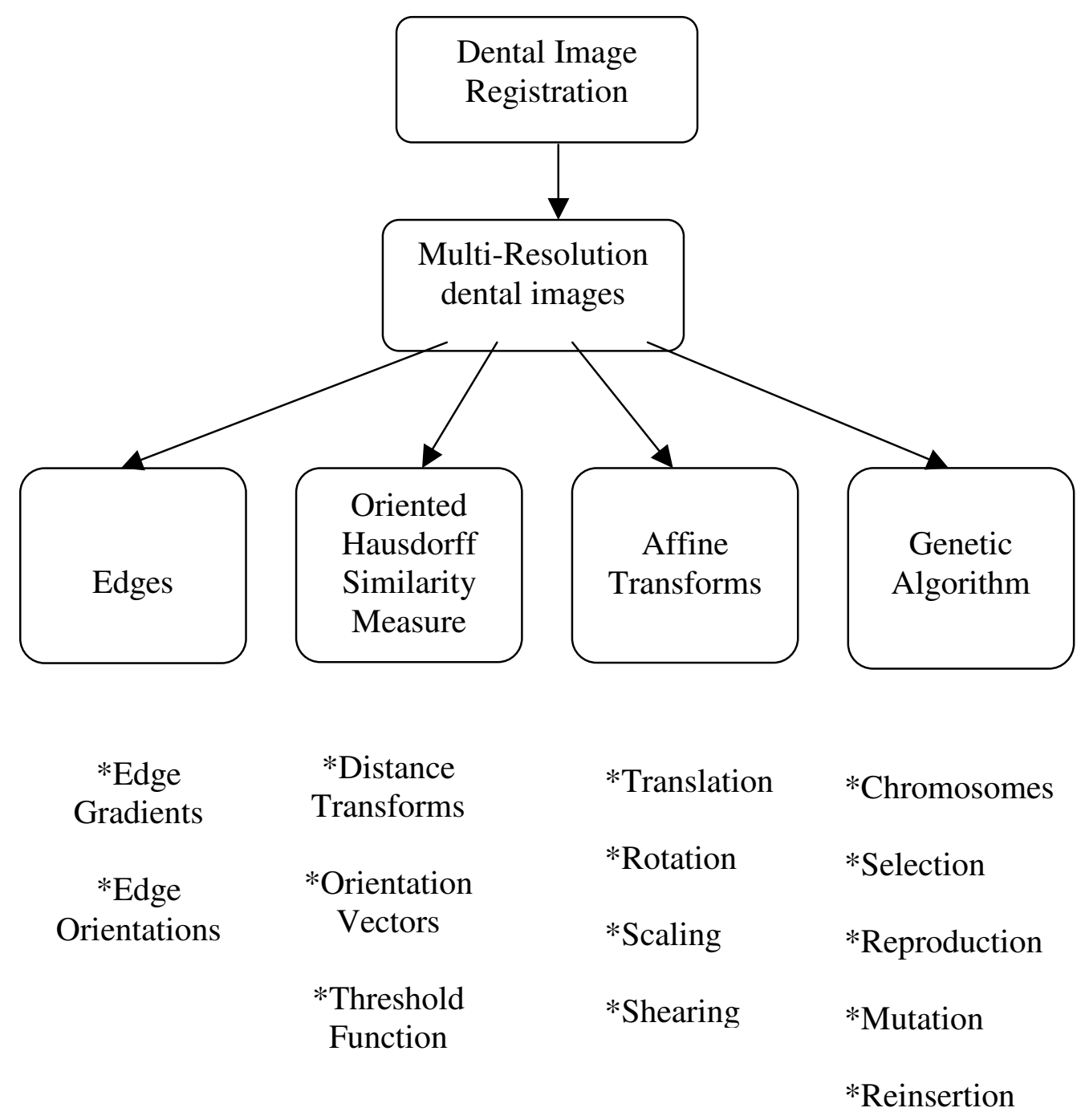

Figure 4.2 Elements of Dental Image Registration 


\subsection{EDGE FEATURE EXTRACTION}

The primary step required to accomplish registering images is to perform feature extraction on reference and query images. We employed edges and edge gradients as features that need to be matched. To retrieve the edge and gradient points of the subject and reference images we employed the following procedure.

Step1: Applied sobel edge operator on image regions with certain threshold value to obtain edge points.

Step2: Created gradient masks using sobel edge emphasizing filters.

Step3: Applied these masks to get gradients of image in both horizontal and vertical directions.

Step4: Calculated the magnitude of gradient from which unit gradient magnitudes of the images are computed.

Considering the reference and subject regions of interest as $\mathrm{P}$ and $\mathrm{Q}$, the edge, gradient and unit gradient images computed are termed as

$\mathrm{P}_{\mathrm{E}} \& \mathrm{Q}_{\mathrm{E}}=$ Edge images of $\mathrm{P} \& \mathrm{Q}$ respectively

$\mathrm{P}_{\mathrm{G}} \& \mathrm{Q}_{\mathrm{G}}=$ Gradient images of $\mathrm{P} \& \mathrm{Q}$ respectively

$\mathrm{O}_{\mathrm{PG}(\mathrm{p})} \& \mathrm{O}_{\mathrm{QG}(\mathrm{p})}=$ Unit gradient vectors of Image $\mathrm{P}$ at position $\mathrm{p}$ and Image $\mathrm{Q}$ at position $\mathrm{q}$.

Figure below shows the edge and gradient dental regions of interest. 

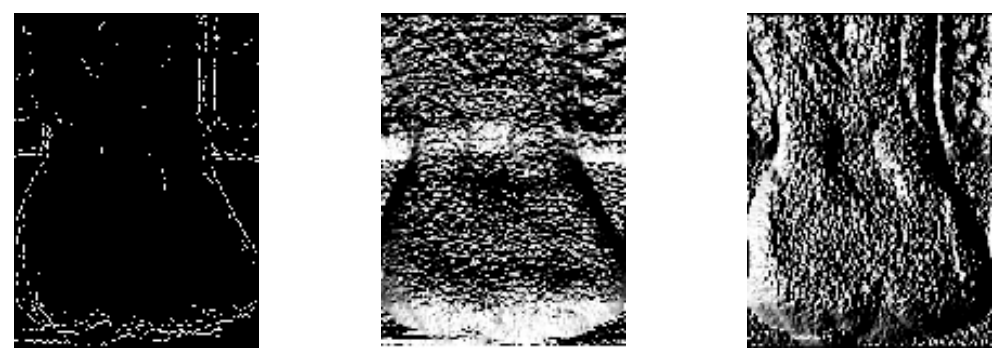

Figure 4.3 Edge and Gradient Dental ROI

\subsection{AFFINE TRANSFORMATIONS}

After the features are segmented from the data sets, a geometric transformation is essential to align the pixels representing these corresponding features, in the subject and reference pair of images. These transformations consist of changes in translation, rotation, scale and shear in order to describe the variation of input image from the reference image.

As elucidated in the Literature review, an affine transformation maps variables in input image to new variables in output image by applying a linear combination of translation, rotation, scaling, and/or shearing. It does not necessarily preserve angles or lengths.

Considering $\left(\mathrm{x}_{1}, \mathrm{y}_{1}\right)$ as coordinate points of subject dental region of interest, $\left(\mathrm{x}_{2}, \mathrm{y}_{2}\right)$ as points of the transformed version of dental ROI, the equation of an affine transformation in the matrix form is: 


$$
\left(\begin{array}{l}
x_{2} \\
y_{2} \\
1
\end{array}\right)=\left(\begin{array}{lll}
a & b & e \\
c & d & f \\
0 & 0 & 1
\end{array}\right) \quad\left(\begin{array}{l}
x_{1} \\
y_{1} \\
1
\end{array}\right)
$$

Eqn (4.1)

The equation is expressed linearly as:

$$
\begin{aligned}
& x_{2}=a x_{1}+b y_{1}+e \\
& y_{2}=c x_{1}+d y_{1}+f
\end{aligned}
$$

The base coordinates $\left(\mathrm{x}_{1}, \mathrm{y}_{1}\right)$ are transformed to produce derived coordinates $\left(\mathrm{x}_{2}, \mathrm{y}_{2}\right)$. The six constants ' $a$ ' through ' $\mathrm{f}$ ' determine the effect of transformation, as follows:

'a' performs scaling or stretching along $\mathrm{X}$ axis.

'b' performs rotation or skewing along $\mathrm{X}$ axis.

'c' performs scaling or stretching along Y axis.

'd' performs rotation or skewing along Y axis.

'e' performs shifting along $\mathrm{X}$ axis.

'f' performs shifting along $\mathrm{Y}$ axis.

Considering a square, the diagram below depicts translation, rotation, scaling and shearing operations. 


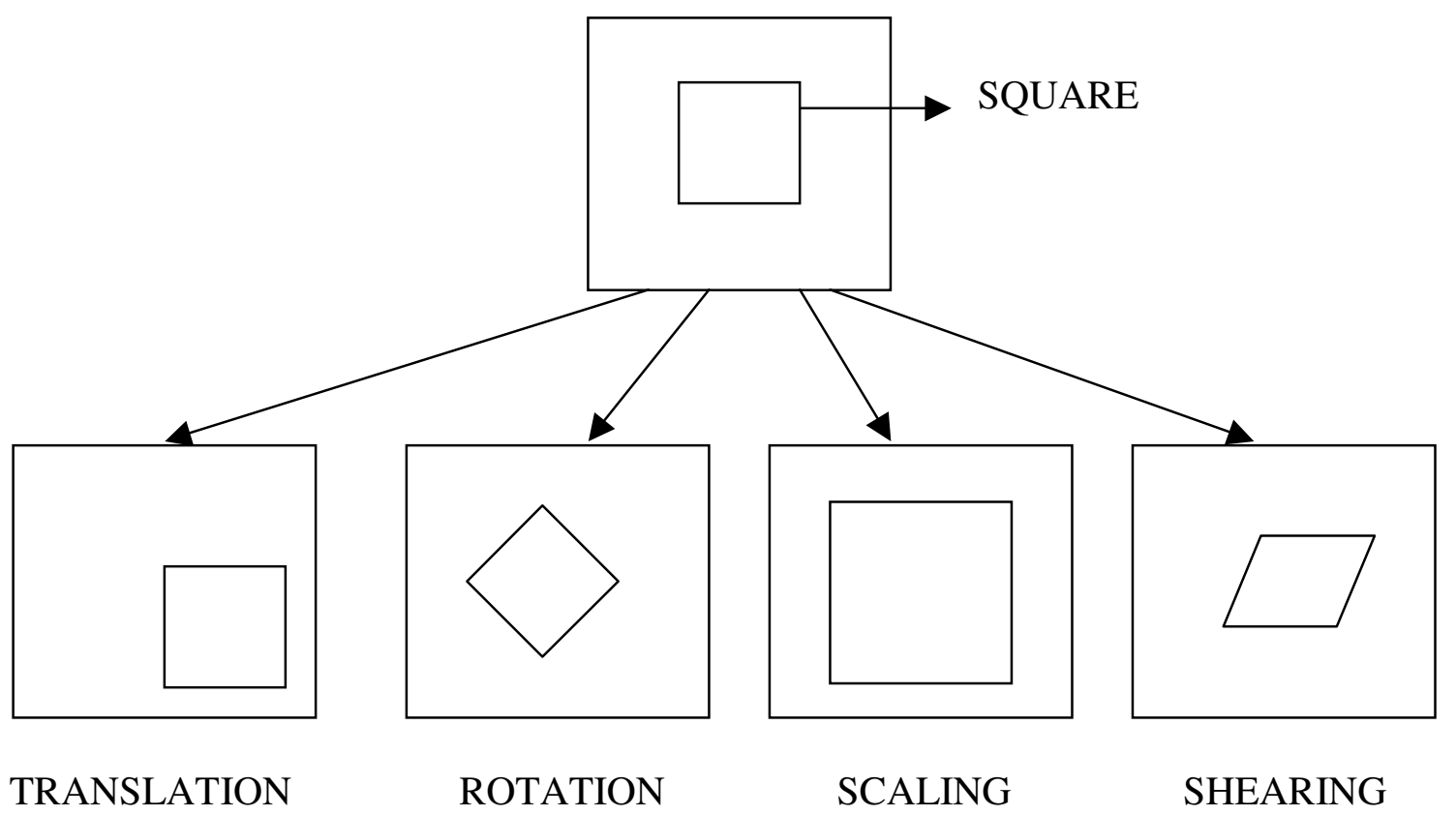

Figure 4.4 Affine Transformations

\subsubsection{BOUNDS ON PARAMETERS OF AFFINE TRANSFORMATION [Nassar05]}

In this section an attempt is made to consider the problem of establishing theoretical bounds on the parameters of affine transformations. We emphasize that such bounds could play a significant role in selecting potential parameters of alignment and search for transformation parameters effectively. We selected the affine transform parameters subject to the bounds discussed below.

We made the following assumptions to derive bounds on the parameters.

a) The area of query image is smaller than that of the reference image.

b) The transformed frame of query image does not strictly cross the boundary of reference, i.e. the transformed query image is totally contained within the area of reference image. 
c) There is no excessive squeezing on the transformed area and no partial occlusion.

Given a reference image I bounded by a rectangular area of height ' $\mathrm{H}$ ' and width ' $\mathrm{W}$ ', and a query image $Q$ bounded by a rectangular region of height ' $h$ ' and width ' $w$ ', we write the bounds on the affine parameters subject to the assumption (b) discussed above

$$
\begin{aligned}
& |\mathrm{a}| \leq \mathrm{H} /(\mathrm{h}-1) \\
& |\mathrm{b}| \leq 2(\mathrm{H}-|\mathrm{a}|(\mathrm{h}-1)) /(\mathrm{w}-1)
\end{aligned}
$$

Similar analysis is done to determine the bounds on parameters c, $\mathrm{d}$ as follows:

$$
\begin{aligned}
& |\mathrm{c}| \leq \mathrm{W} /(\mathrm{h}-1), \\
& |\mathrm{d}| \leq 2\left(\mathrm{~W}-\left.\right|_{\mathrm{c}} \mid(\mathrm{h}-1)\right) /(\mathrm{W}-1)
\end{aligned}
$$

We determine the extent of the transformed query image without translation as follows:

$$
\begin{aligned}
& X_{\min }=\min \{a+b, a+b w, a h+b w, a h+b\} \\
& X_{\max }=\max \{a+b, a+b w, a h+b w, a h+b\} \\
& Y \min =\min \{c+d, c+d w, c h+d w, c h+d\} \\
& Y_{\max }=\max \{c+d, c+d w, c h+d w, c h+d\}
\end{aligned}
$$

Thus the bounds on the translation parameters e and $\mathrm{f}$ can be determined with the aid of above equations as follows:

$$
\begin{aligned}
& 1-\delta_{\mathrm{tx}}-\mathrm{X}_{\min } \leq \mathrm{e} \leq \mathrm{H}+\delta_{\mathrm{tx}}-\mathrm{X}_{\max } \\
& 1-\delta_{\mathrm{ty}}-\mathrm{Y}_{\min } \leq \mathrm{f} \leq \mathrm{W}+\delta_{\mathrm{ty}}-\mathrm{Y}_{\max }
\end{aligned}
$$

$\delta_{\mathrm{tx}}$ and $\delta_{\mathrm{ty}}$ account for the requirement of overlap between the query and reference objects. 


\subsection{SIMILARITY MEASURE}

Determining similarity between reference and subject images is the next vital issue in our registration process. We employed oriented Hausdorff measure to compute similarity between reference and transformed version of the input image. This method is based on Hausdorff similarity measure using distance map values. The similarity measure consists of the following components.
a) Hausdorff measure
b) Distance transform map
c) Threshold function

We present a brief description of Hausdorff similarity measure, Distance transform maps and threshold function below.

\subsubsection{HAUSDORFF SIMILARITY MEASURE [Huttenlocher93]}

The generalized Hausdorff measure provides a means of determining the resemblance of one point set to another, by examining the fraction of points in one set that lie near points in the other set. This distance is used to find the degree of resemblance between two objects that are superimposed on one another.

Considering two finite sets of points $\mathrm{P}=\left\{\mathrm{p}_{1}, \mathrm{p}_{2} \ldots \mathrm{p}_{\mathrm{n}}\right\}$ and $\mathrm{Q}=\left(\mathrm{q}_{1}, \mathrm{q}_{2} \ldots \mathrm{q}_{\mathrm{n}}\right\}$ the Hausdorff distance $\mathrm{H}$ is defined as

$$
\mathrm{H}(\mathrm{P}, \mathrm{Q})=\max (\mathrm{h}(\mathrm{P}, \mathrm{Q}), \mathrm{h}(\mathrm{Q}, \mathrm{P}))
$$

Where $\mathrm{h}(\mathrm{P}, \mathrm{Q})=\max \min \|\mathrm{p}-\mathrm{q}\|$

$$
\mathrm{p} € \mathrm{P} q € Q
$$

where $\|$.$\| is Euclidean norm on the points of \mathrm{P}$ and $\mathrm{Q}$. 
$\mathrm{h}(\mathrm{P}, \mathrm{Q})$ reflects the distance of the point of $\mathrm{P}$ that is farthest from any point of $\mathrm{Q}$ using the given Euclidean norm. Each point of $\mathrm{P}$ is ranked based on its distance to the nearest point in $\mathrm{Q}$ and the largest ranked point is considered as the most mismatched point. Thus the maximum of $h(P, Q)$ and $h(Q, P)$ gives the degree of mismatch between both the sets [Huttenlocher93].

\subsubsection{DISTANCE TRANSFORM MAP}

Traditionally, a Distance Transformation is defined as an operation that transforms a binary digital image consisting of feature and non-feature pixels into a distance map where all non-feature pixels have a value corresponding to a measure of distance from it to the nearest feature pixel.

We determined the Euclidean distance transform of an edge image. For each pixel in the distance map the distance transform assigns a number that is the distance between that pixel and the nearest non zero pixel of the edge image.

In 2-D, the Euclidean distance between $\mathrm{P}_{1}=\left(\mathrm{x}_{1}, \mathrm{y}_{1}\right)$ and $\mathrm{P}_{2}=\left(\mathrm{x}_{2}, \mathrm{y}_{2}\right)$ is:

$\mathrm{D}_{\mathrm{E}}\left(\mathrm{P}_{1}, \mathrm{P}_{2}\right)=\sqrt{ }\left(\mathrm{x}_{1}-\mathrm{x}_{2}\right)^{2}+\left(\mathrm{y}_{1}-\mathrm{y}_{2}\right)^{2}$

Thus the distance transform map of image $Q$ at position $p$ is termed as $d_{Q}(p)$ and is given

by $\mathrm{d}_{\mathrm{Q}}(\mathrm{p})=\min \|\mathrm{p}-\mathrm{q}\|$

$\operatorname{Eqn}(4.8)$

q€Q

Figure 4.5 Distance transform map of Dental ROI 


\subsubsection{THRESHOLD FUNCTION}

The similarity function of the algorithm has a threshold function shown below indicating that the value of the function reaches it maximum at some values of the transform and is zero other wise. Thus the distance transform values that are between negative and positive value of threshold are considered for the evaluation of $\rho_{T}\left(d_{Q}(p)\right)$. Hence the governing equation here is

$\mathrm{y}=-\left((\mathrm{x} / \mathrm{T})^{2}-1\right)$; where $\mathrm{y}=\rho_{\mathrm{T}}\left(\mathrm{d}_{\mathrm{Q}}(\mathrm{p})\right) ; \mathrm{x}=\mathrm{d}_{\mathrm{Q}}(\mathrm{p})$ and positive $\mathrm{T}$ is the threshold value. Following figure shows the threshold function.

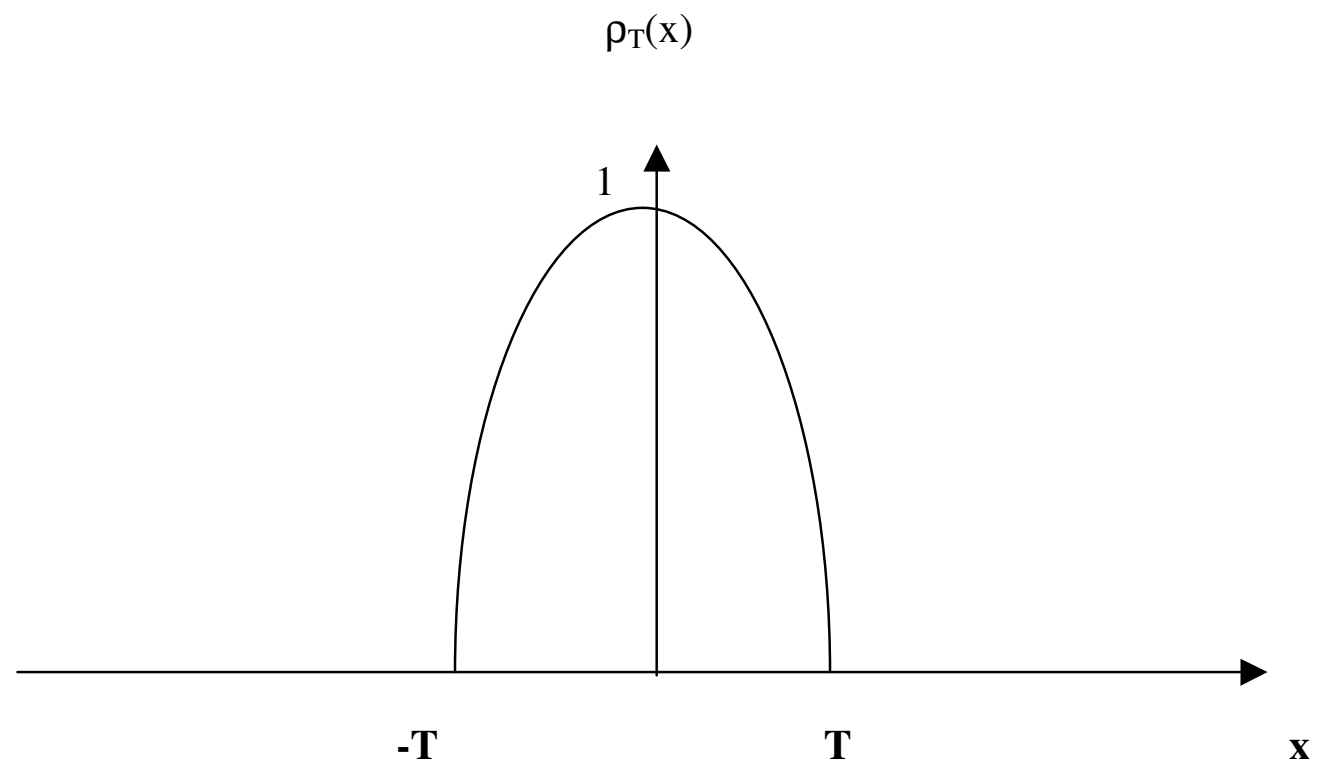

Figure 4.6 Similarity function of the algorithm

\subsubsection{SIMILARITY MEASURE COMPUTATION [Sim01]}

The directed Oriented Hausdorff Similarity's using these edge, gradient distance transform maps and threshold function is determined as below. 
The directed OHS is defined as

OHS $\left(\mathrm{P}_{\mathrm{G}}, \mathrm{P}_{\mathrm{E}}, \mathrm{d}_{\mathrm{Q}}\right)=\Sigma_{\mathrm{p} \in \mathrm{PE}} \mathrm{O}_{\mathrm{PG}(\mathrm{p})} . \mathrm{O}_{\mathrm{QG}(\mathrm{p})} \rho \mathrm{T}\left(\mathrm{d}_{\mathrm{Q}}(\mathrm{p})\right)$

$$
=\Sigma_{\mathrm{p} \in \mathrm{P}} \mathrm{s}(\mathrm{p}) \cdot \rho \mathrm{T}\left(\mathrm{d}_{\mathrm{Q}}(\mathrm{p})\right)
$$

$\mathrm{s}(\mathrm{p})=\mathrm{O}_{\mathrm{PG}(\mathrm{p})} . \mathrm{O}_{\mathrm{QG}(\mathrm{p})}$, dot product of $\mathrm{O}_{\mathrm{PG}(\mathrm{p})} \& \mathrm{O}_{\mathrm{QG}(\mathrm{p})}$

$\rho T(x)$ is a symmetric threshold function and is defined as shown above.

$\left(\mathrm{d}_{\mathrm{Q}}(\mathrm{p})\right)=$ Distance map value of Edge Image of $\mathrm{Q}$ at position $\mathrm{p}$

OHS $\left(Q_{G}, Q_{E}, d_{P}\right)$ is calculated in a similar way.

Similarity measure is the minimum of two directed Oriented Hausdorff Similarity's.

$\mathrm{H}=\min \left(\mathrm{OHS}\left(\mathrm{P}_{\mathrm{G}}, \mathrm{P}_{\mathrm{E}}, \mathrm{d}_{\mathrm{Q}}\right), \mathrm{OHS}\left(\mathrm{Q}_{\mathrm{G}}, \mathrm{Q}_{\mathrm{E}}, \mathrm{d}_{\mathrm{P}}\right)\right)$

Eqn (4.10)

The algorithm is derived by embedding robust Hausdorff measure into accumulating operation of Hough Transform structure. The algorithm is derived from Hausdorff measure for determining similarity using the distance transform maps (Euclidean norm on images $\mathrm{P}$ and $\mathrm{Q})$. The similarity is calculated by accumulating the distance value of distance transform map and orientation information [Sim01]. Also the algorithm uses minimum operator to find the similarity measure unlike Hausdorff conventional algorithm that uses maximum operator. Using maximum operator may give large incorrect distances and determines the mismatch between objects. The algorithm takes into account the orientation of pixels while accumulating the distance values, which aids

in eliminating wrong correspondences. Thus the algorithm is proved to be robust to severe noise, degradations and outliers which are anomalous data far from assumed error distribution. [Sim01].

\subsection{SEARCH STRATEGY - GENETIC ALGORITHM}

We have applied Genetic algorithm as a search strategy to reduce the search space for registration of dental ROI's. The algorithm is an intelligent way of exploiting the search 
in a random manner within a defined search space to obtain a solution thus solving the problem. In the process of using GA, finding solutions generates other points (possible solutions) as evolution proceeds.

\subsubsection{SEARCH USING GENETIC ALGORITHM}

The Algorithm initially starts considering a number of possible solutions that represent the chromosomes. The chromosome string is generally a binary string containing " 0 " and "1". The initial population is taken randomly [Chalermwat99]. Each such solution is an individual population. An initial fitness value is assigned to these individual populations that are based on the ranking of the performance on the individuals over the defined objective function. Based on the fitness values, some candidates with a fitness value above a certain threshold condition are selected for the reproduction in the next generations. The reproduction operation is called crossover. After reproduction the mutation operator is applied to disturb the chromosome to verify that the possibility of searching a particular subspace of the problem is never zero. This process is continued till a suitable termination criterion is reached. It may be limited to number of generations or convergence to the solution. [Chalermwat99]

\subsubsection{COMPONENTS OF GENETIC SEARCH}

The key components of a Genetic Algorithm are as follows:

Chromosomes: Every solution is represented by a chromosome and is typically a binary representation. Real valued representation of the chromosomes is also used.

Objective function: It is used to determine the measure of individual performance for a given problem. In this research similarity measure between the input and reference dental regions of interest plays the role of objective function.

Selection: Selection is a process in which individuals are selected to reproduce, generate off springs for the next generation. Usually, the best individuals (those having higher 
fitness values) will have a greater probability for being selected. The process of selection determines the number of times a particular individual is selected based on a specified method. Roulette Wheel Selection and Stochastic Universal Sampling are the two common selection methods used. We adopted Stochastic Universal Sampling in this thesis. [Chalermwat99]

Reproduction: Reproduction is the recombination between pairs of individuals to return new individuals after mating. We have adopted single point crossover for reproduction in this work.

Mutation: Mutation can be termed as replacement or disturbance of genetic material into the population thus producing the new structure for population.

Reinsertion: After the new population is determined with selection and mutation process their objective function is calculated and their fitness is evaluated. These new individuals that are produced after selection and mutation are again inserted into the population based on their fitness values. Thus it is useful to maintain the size of original population.

\subsubsection{GENETIC ALGORITHM}

We present a simple algorithm depicting the search. [Chalermwat99]

1) Start

2) gen $=0$; // first generation//

3) Initialize Pop (gen)

4) Determine fitness of Pop (gen)

5) While the termination criteria is not reached do \{

6) gen $=$ gen +1 ; // incrementing the generation counter // 
7) Select Pop (gen) from Pop (gen - 1);

8) Reproduce pairs in Pop (gen);

9) Determine fitness Pop (gen); $\quad$ \}

10) End

\subsubsection{STOCHASTIC UNIVERSAL SAMPLING [Gatbx]}

The selection algorithms consist of measures of performance called bias and spread. The absolute difference between an individual's actual and expected selection probability is called bias and the range of possible number of trials the individual is selected is known as spread. Stochastic universal sampling (SUS) algorithm has minimum spread and zero bias. SUS uses $\mathrm{N}$ equally spaced pointers, where $\mathrm{N}$ is the number of selections required. The population is shuffled randomly and a single random number in the range [0 Sum/ $/ \mathrm{N}$ ] is generated 'ptr'. Sum is the sum of individuals' expected selection probabilities or the sum of raw fitness values over all the individuals in the current populations. The $\mathrm{N}$ individuals are then chosen by generating the $\mathrm{N}$ pointers spaced by $1,[\mathrm{ptr}, \mathrm{ptr}+1, \ldots$, ptr $+\mathrm{N}-1]$, and selecting the individuals whose fitness span the positions of the pointers.

\subsubsection{SINGLE POINT CROSSOVER}

In this type of crossover between two individuals, one crossover point is selected, binary string from the beginning of the chromosome to the crossover point is copied from the first parent, the rest is copied from the other parent. This is done according to the specified crossover probability. The pairs are mated in order that is odd row with the next even row and so on. 


\subsection{SEARCH DATA REDUCTION USING MULTI RESOLUTIONS}

We have attempted two achieve search data reduction using multi- resolutions of dental ROI's. They are

a) Image sub-sampling using interpolation

b) Wavelet decomposition

\subsubsection{SUB -SAMPLING USING INTERPOLATION}

Sub-sampling is reduction in the size of an image. It is performed by replacement of a group of pixel values by one arbitrarily chosen pixel value from within this group or by interpolating between pixel values in local neighborhoods.

There are two common approaches to image sub-sampling.

\section{REPLACEMENT OR NEAREST NEIGHBOR INTERPOLATION}

In the first technique, one pixel value within a local neighborhood that is nearest neighbor is chosen to be a representative of its surroundings and it replaces the group of pixels to shrink the size of an image. This method is computationally simple.

\section{BILINEAR AND BICUBIC INTERPOLATION}

The second method interpolates between pixel values within a neighborhood by taking a statistical sample such as mean of local intensity values. For bilinear interpolation, the output pixel value is a weighted average of pixels in the nearest 2-by-2 neighborhood. For bi-cubic interpolation, the output pixel value is a weighted average of pixels in the nearest 4-by-4 neighborhood

We have adopted nearest neighboring method to sub-sample our reference and subject dental ROI' s.

We have compressed the reference and subject dental ROI's of size $\mathrm{P} * \mathrm{Q}$ to $\mathrm{P} / 2 * \mathrm{Q} / 2$. They are compressed further to obtain multi-resolutions of input and reference images in 
lower levels. At every level the image is compressed to one fourth the size of the previous level. The figures below show the sub sampling process.

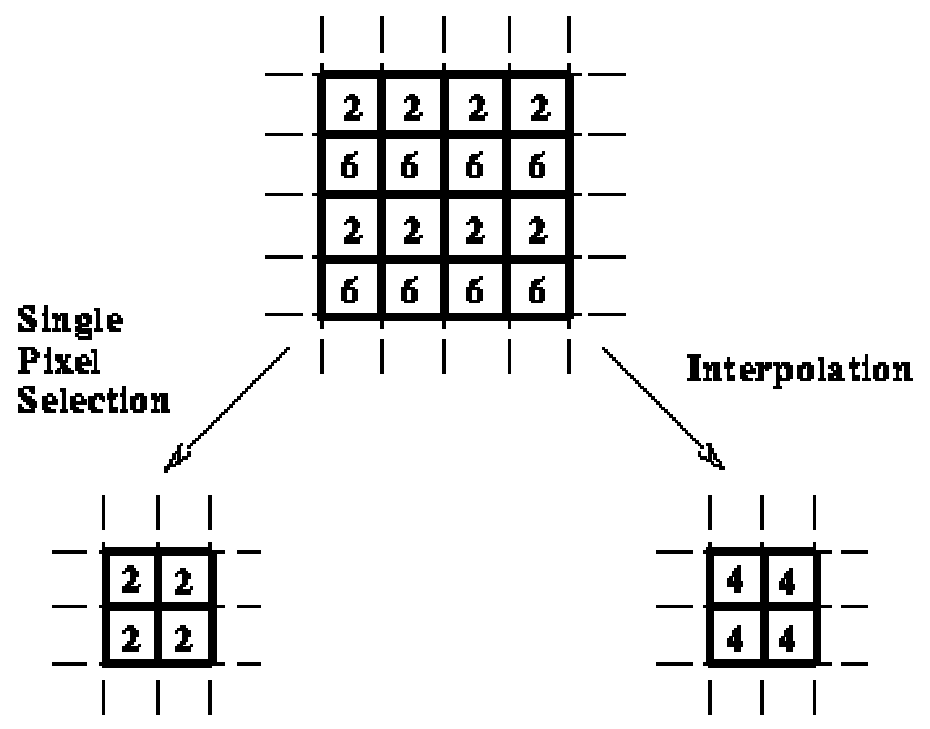

Figure 4.7 Methods of sub sampling a) Replacement using upper pixel b) Interpolation using mean value
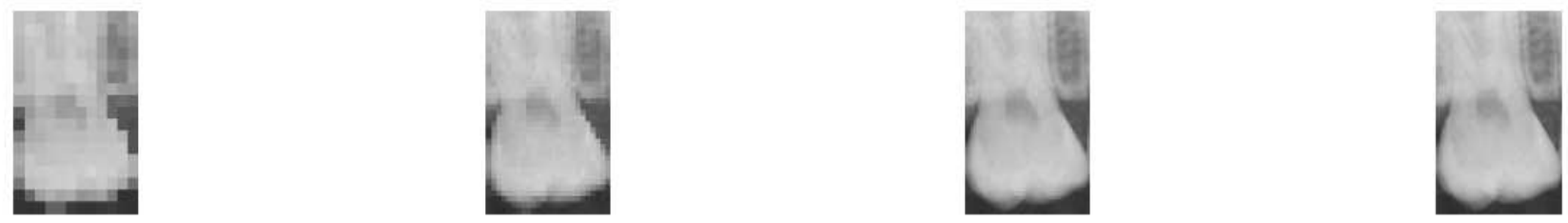

Figure 4.8 Multi-resolution Dental ROI's using sub-sampling with nearest neighbor interpolation 


\subsubsection{WAVELET DECOMPOSITION}

The second method we have attempted to achieve a reduction in the search data is using multi-resolution property of the wavelets. In this technique multi-resolution property of wavelets is used to decompose the image from coarse to finer levels.

A Wavelet transform is capable of providing time and frequency information simultaneously, hence giving a time-frequency representation of the signal. The wavelet decomposition of an image of size $\mathrm{P} * \mathrm{Q}$ produces 4 images each of size $\mathrm{P} / 2 * \mathrm{Q} / 2$. The size of these images is reduced to $1 / 4$ of the original size [Chalermwat99]. They are LL, LH, HL, and LL.

The images are named based on the filter (high pass or low pass) applied to the image in horizontal and vertical directions [Chalermwat99]. The LL image contains all the details of the original image and is a reduced version of the original image. The LH version contains the horizontal edges and the HL contains vertical edges. The $\mathrm{HH}$ version contains the high frequency edges and noise. We have selected this LL version of the image to produce next level of decomposition.

The flow diagrams in the figure 4.9 and dental regions of interest in figure 4.10 depict the wavelet decomposition. 


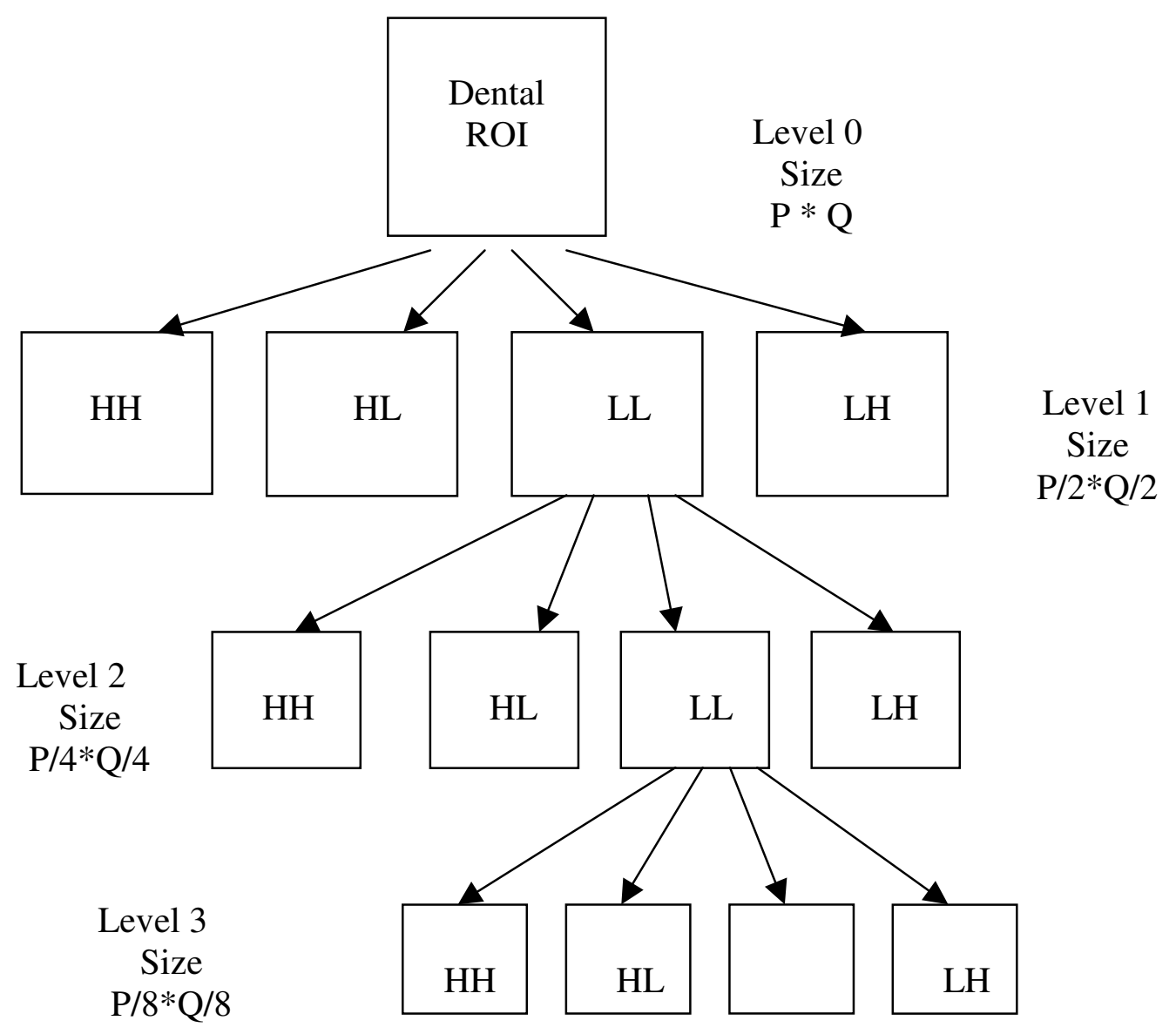

Figure 4.9 Levels of wavelet Decomposition
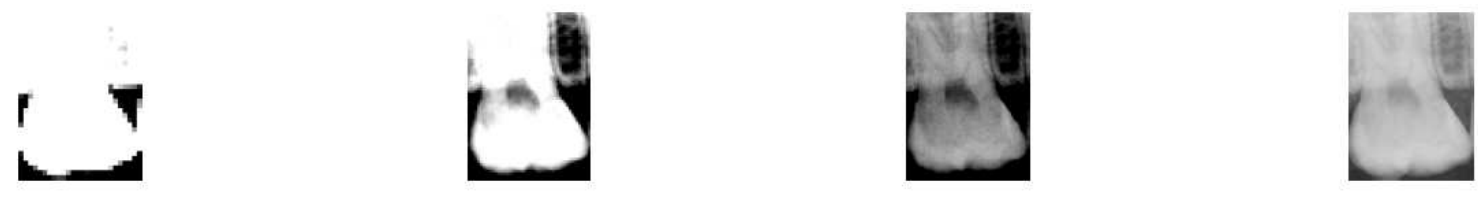

Figure 4.10 Multi Resolution Dental ROI's using wavelet decomposition

The block diagram shown in figure 4.11 depicts the process flow of implemented image registration technique. 


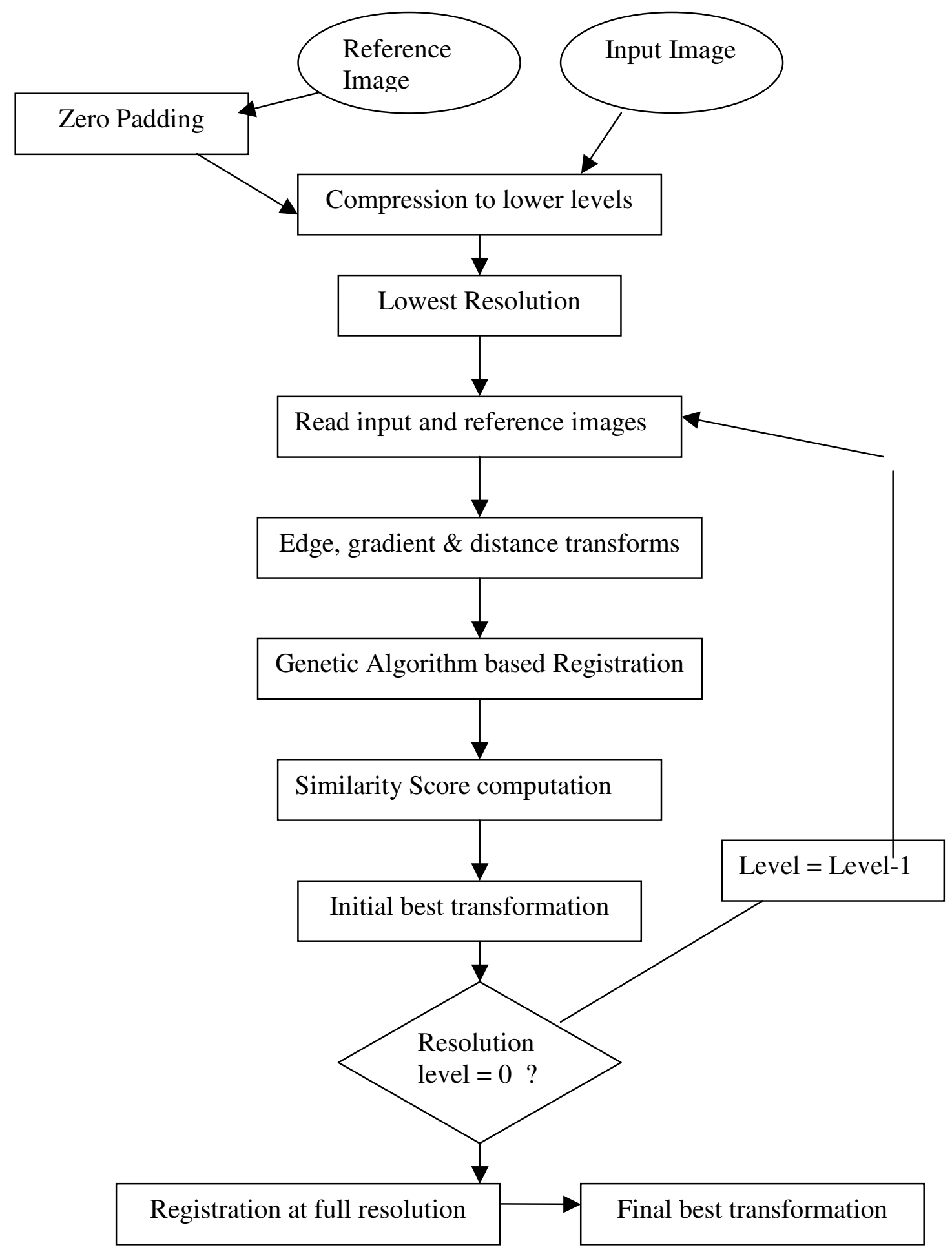

Figure 4.11 Block Diagram of Dental Image Registration 


\subsection{THE REGISTRATION TECHNIQUE}

We have combined the Genetic algorithm search and multi resolution approach. Initial image registration is carried out at the lowest resolution level. The following steps depict the process of registration

a) Computed the edge, gradient images and distance transform maps of both the input and reference dental regions of interest for the evaluation of Oriented Hausdorff similarity measure.

b) The reference edge image is zero-padded by half of its size both height wise and width wise to allow for tolerance of transformations when the transformed subject image is superimposed onto the reference image. The zero pad image is shown below.

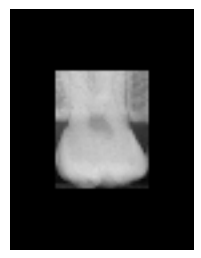

Figure 4.12 Zero padded Reference Image

c) We created an initial population in the lowest resolution that consists of chromosomes for the genetic search algorithm. The chromosome string is generally a binary string containing " 0 " and " 1 ". The initial population is taken randomly. We have considered binary and real valued representations. 
d) Each chromosome is an affine transformation parameter (a, b, c, d, e, and f). These chromosomes are created in a way such that they are within their bounds as described in section 4.3.1. Each such set of parameters is an individual population.

e) Then we transform the query image using these transformation parameters and similarity is computed between every transformation of the query image and the reference dental region of interest. The oriented Hausdorff similarity function thus plays the role of objective function in the search algorithm.

f) The individuals that give higher similarity measures between are considered as the best ones and are ranked based on their similarity measures.

g) A certain threshold on the population that is to be selected for further fitness evaluation. The individuals that are above the threshold are selected using a selection criterion. We have adopted the stochastic universal sampling criteria for the selection basis.

h) These selected individuals are recombined among themselves using a single point crossover criterion with a selected crossover rate. We also utilize the mutation operator on the chromosomes so that there is an exchange in the chromosomes of the pair of individuals for better results.

i) Once the new population has been generated the query image is transformed using these chromosome values that act as the affine transformation parameters and fitness is evaluated (i.e. similarity score is computed between the reference and the query image).

j) We reinsert these new population into the initial population based on their ranking of similarity score to maintain the size of the original population. This process of selection, reproduction, mutation, reinsertion are carried out through specified number of generations till the transformed input image aligns with the reference and the similarity score obtained becomes stabilized i.e. constant.

k) We plot a graph between the maximum similarity and the number of generations. The genetic algorithm is terminated after reaching some specified number of generations as the similarity graph becomes constant/non-increasing. The graph is shown below. 


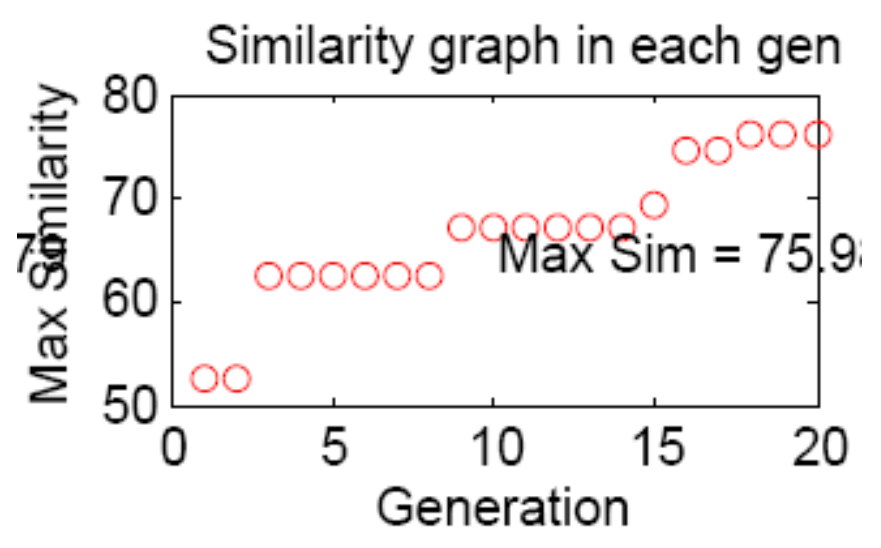

Figure 4.13 Graph of similarity measure and generations

\subsubsection{REGISTRATION IN FINER LEVELS}

The resultant transformation parameters in the lower level that give maximum similarity are updated to the finer levels thus forming the basis for finer level registration process i.e. the best-found transformations in the coarser level are considered as the population for the next level. In addition to these some arbitrary population are created in every higher resolution level to maintain the size of original population. The registration process is carried out as elucidated for the lowest resolution with the genetic search algorithm components and the transformations are updated to the finer levels till the registration is carried out in the zero level i.e. (level where the size of the dental ROI is $\mathrm{P} * \mathrm{Q})$. The final transformation parameters that give the highest similarity score are recorded. Figure 4.14 and 4.15 depict the registration technique. 

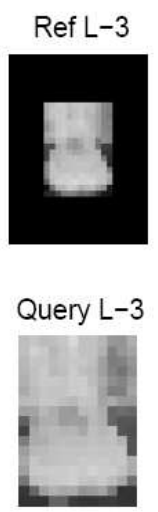

Registered L-3

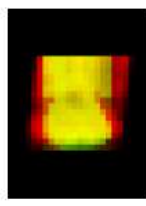

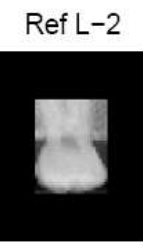

Query L-2

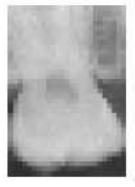

Registered L-2

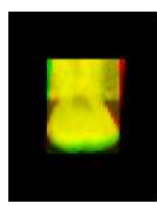

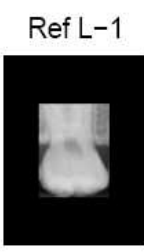

Query L-1

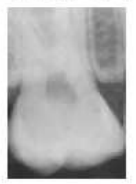

Registered L-1

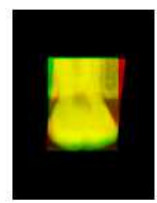

Ref $L-0$

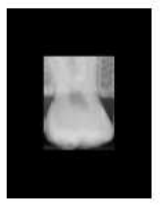

Query L-0

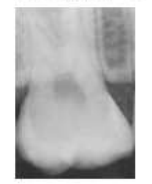

Registered L-0

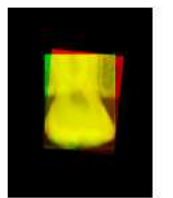

Figure 4.14 Registration of Dental ROI's using multi resolution sub-sampling

Ref $L-3$

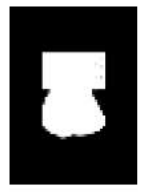

Query L-3

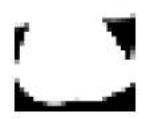

Registered L-3

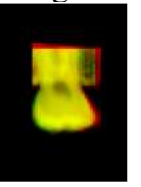

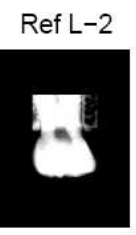

Query L-2

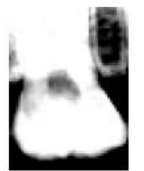

Registered L-2

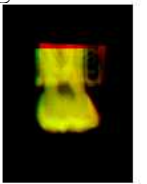

Ref $L-1$

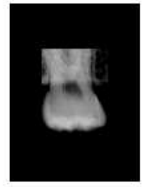

Query L-1

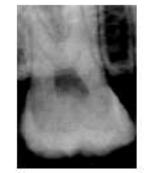

Registered L-1

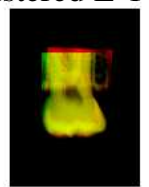

Ref $L-0$

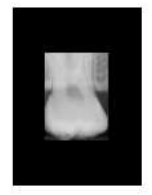

Query L-0

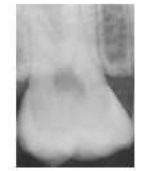

Registered L-0

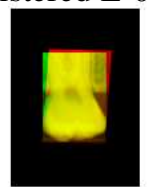

Figure 4.15 Registration of Dental ROI's using multi resolution wave let decomposition 


\subsubsection{TERMINATION CRITERIA}

As we move across the levels of image resolutions the number of generations are reduced by half that of the previous resolution. Thus the termination criterion is the number of generations after which the similarity score graph becomes constant. We have also specified a threshold on the time consumed for registration, so that the algorithm is terminated within the given time and the transformation parameters are recorded, irrespective of the resolution level in which the registration is being carried out. The determining termination criterion is the one that occurs first between the number of generations and the time limit.

The figures below present an illustration of the termination criteria. Figure a) represents the Reference and Query images. The figure b) represents the registered output in the lowest resolution level and figure c illustrates the graph between similarity score and the generations with a 10 minute time limit.

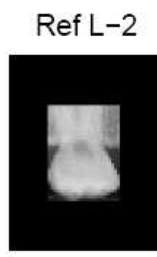

Query L-2

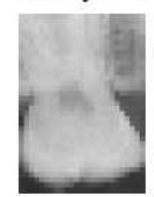

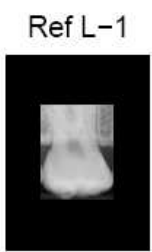

Query L-1

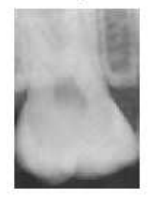

Ref L-0

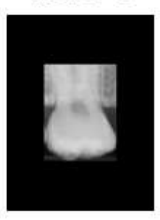

Query L-0

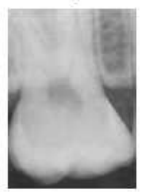

Figure 4.16 a) Reference and Query Dental ROI's 


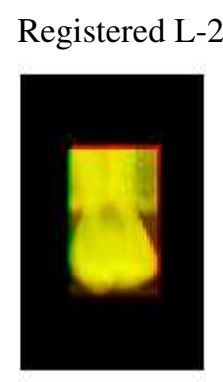

Figure 4.16 b) Registered Dental ROI in lower resolution level with a ten minute limit

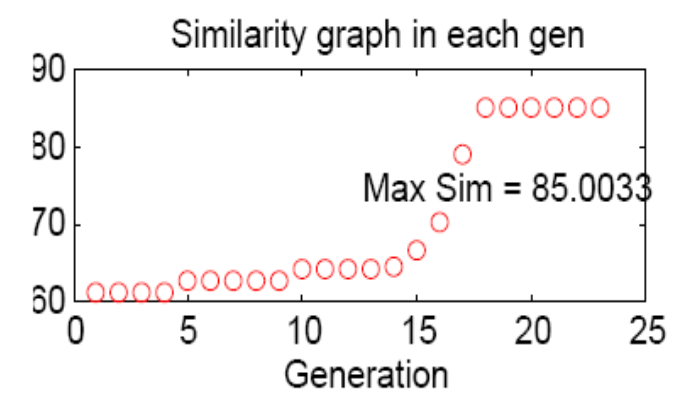

Figure 4.16 c) Graph of similarity measure and generations with a ten minute limit

\subsection{ADVANTAGES OF MULTI-RESOLUTION GENETIC SEARCH}

a) Using multi resolution approach based on genetic algorithm for registration effectively reduced search space and search data.

b) We obtain a reduction in the search data as we performed initial registration at the lowest resolution where the image size is very much reduced compared to the size of original dental ROI.

c) Since the least fit chromosomes (i.e. the affine parameters) in the lowest resolution are eliminated, we obtain a reduction in the search space as we proceed to higher levels using the genetic algorithm along with multi resolutions approach. GA's proved useful and efficient when search space is large and complex. 
d) There was a reduction in the time required for computation of registration parameters over the linear search methods.

\subsubsection{PARAMETERS OF THE APPROACH}

a) A population size of 700 to 900 suited the considered dental regions of interest.

b) Sobel edge operator has been used with a threshold value of 0.025 for edge detection.

c) Wavelet decomposition of dental regions of interest was done using Haar filter with threshold value increasing above 1.185 in every level of resolution.

d) A threshold has been imposed on the size of the lowest resolution image above a certain value i. e above $(20 * 20)$.

e) We selected Euclidean distance transforms for finding the distance between the pixels.

f) A threshold value of 4 has been set for the threshold function used for evaluating similarity

g) Considered a generation gap of 0.9 and $60 \%$ crossover for reproduction in the genetic search.

h) Adopted stochastic universal sampling for the selection of chromosomes and single point crossover method for reproduction.

i) Considered 40 generations in the lowest resolution level and decreased them by half the value as we proceed to higher levels of resolutions.

j) We place a limitation on the time consumed for the total registration process. The algorithm we developed is suitable to terminate within a specified time limit. 


\section{CHAPTER 5}

\section{RESULTS AND ANALYSIS}

In this chapter we focus on the results obtained by testing our approach for dental image registration. We have adopted testing using two methodologies, one using known transformation parameters and the other using unknown transformation parameters. In Section 5.1 we describe testing using unknown transformation parameters, while section 5.2 deals with testing using known parameters.

The registration component takes segmented regions of dental images that are obtained after the segmentation stage as inputs for registration. The image alignment approach we developed aligns the subject dental region of interest to the reference dental region of interest. The Ante mortem and Post mortem dental regions of interest are used as the subject and reference images interchangeably.

We have implemented our approach on Pentium 1V processor, $512 \mathrm{MB}$ RAM, 1.6 GHz PC. We adopted MATLAB programming for the code functions required for the registration technique. The genetic algorithm functions have been imported from Genetic Algorithm Toolbox [Gatbx].

The testing algorithm involves data sets. One named synthetic data set that corresponds to images for which we know the transformation parameters and the other known as genuine data sets that contain images with unknown values of transformation. We also evaluate the method based on the subjective and objective measures. Subjective measure measures accuracy by visual inspection where as objective measure uses statistical metrics for evaluation [Millwala04]. 


\subsection{TESTING WITH UNKNOWN TRANSFORMATION PARAMETERS}

This is one of methods used for testing. We implement registration and obtain final transformation parameters. We then apply these transformations on the query image to obtain a transformed query image. Then we use subjective methods to verify the accuracy of registered pair. The measure consists of visual inspection to assess whether the points of the query image have been mapped to that of the reference image. Then we calculate the root mean square error and Signal to noise ratio on the subject and reference images.

Considering $f_{1}(x, y)$ as the coordinates for reference image and $f_{2}(x, y)$ as coordinates of query image and respective image sizes as $\mathrm{A}$ and $\mathrm{B}$, we evaluate the root mean square error (RMSE) and signal to noise ratio (SNR) [Gonza02] as:

$$
\begin{aligned}
& \operatorname{RMSE}=\left(1 / M N \sum_{x=0}^{M-1} \sum_{y=0}^{N-1}\left[f^{\prime}(x, y)-f(x, y)\right] 2\right)^{1 / 2} \\
& \operatorname{SNR}=\frac{\sum_{x=0}^{M-1} \sum_{y=0}^{N-1} f^{\prime}(x, y) 2}{\sum_{x=0}^{M-1} \sum_{y=0}^{N-1}\left[f^{\prime}(x, y)-f(x, y)\right] 2}
\end{aligned}
$$

The block diagram showing the process flow of testing using unknown transformation parameters is as follows: 


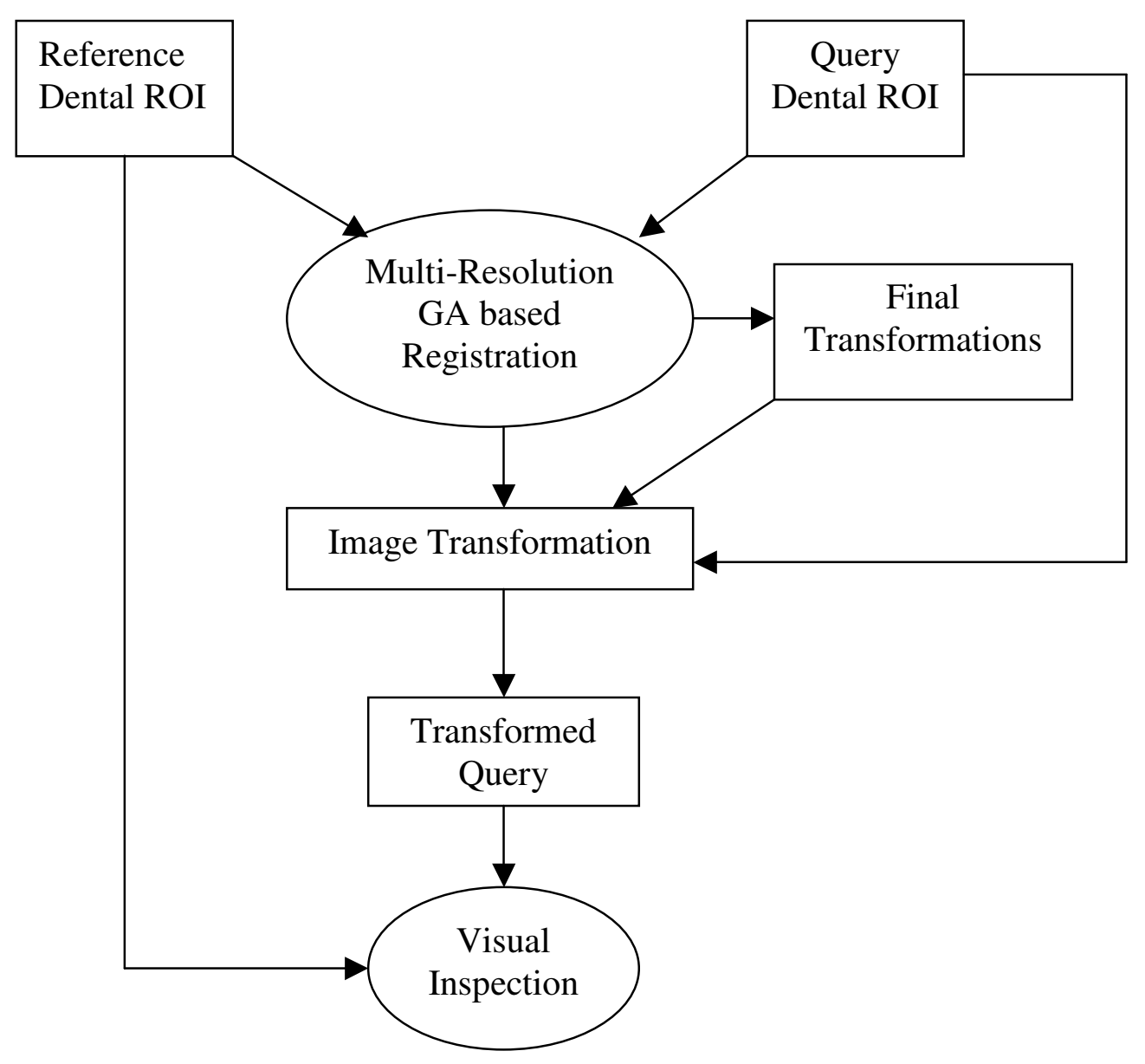

Figure 5.1 Block Diagram for testing using unknown transformation 
Figures below depict the plot of RMSE and Signal to Noise ratios for registered ROI's.
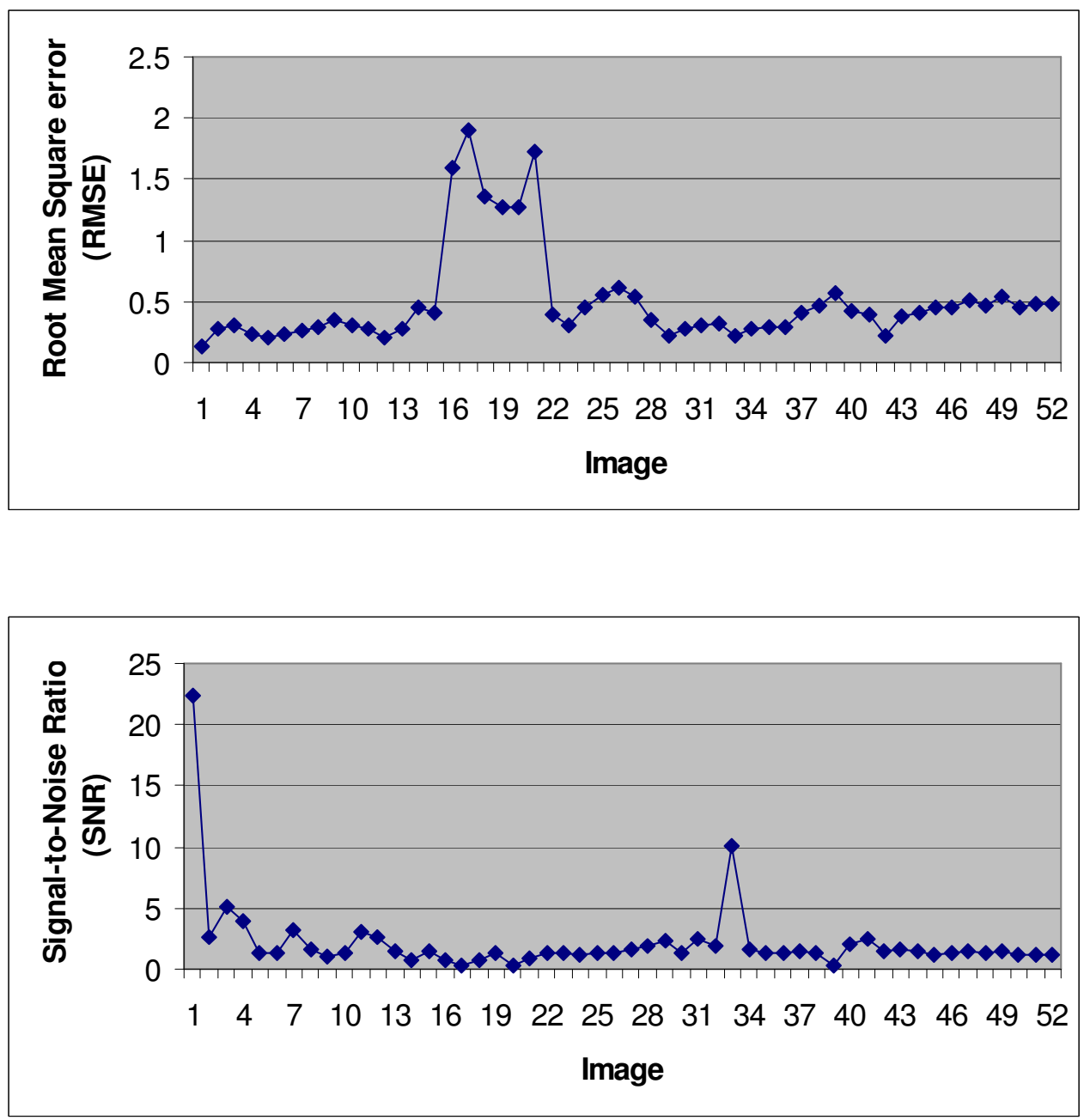

Figure 5.2 Plots of RMSE and SNR for Dental ROI's

The low RMSE values between the subject and reference images from the plots in figure 5.2 indicate good performance in accuracy level. Test cases that are heavily misaligned show increase in the RMSE values (e.g. images from 13 to 22). Signal to Noise ratio is a measure to determine the extent of noise present in images. The low Signal to noise ratio in most cases indicates high noise distributed over the entire test dental images. 
The following are the plots for the termination criteria using 3, 5, 10 and 15 minutes run times for the RMSE and SNR between the images for unknown parameter testing.
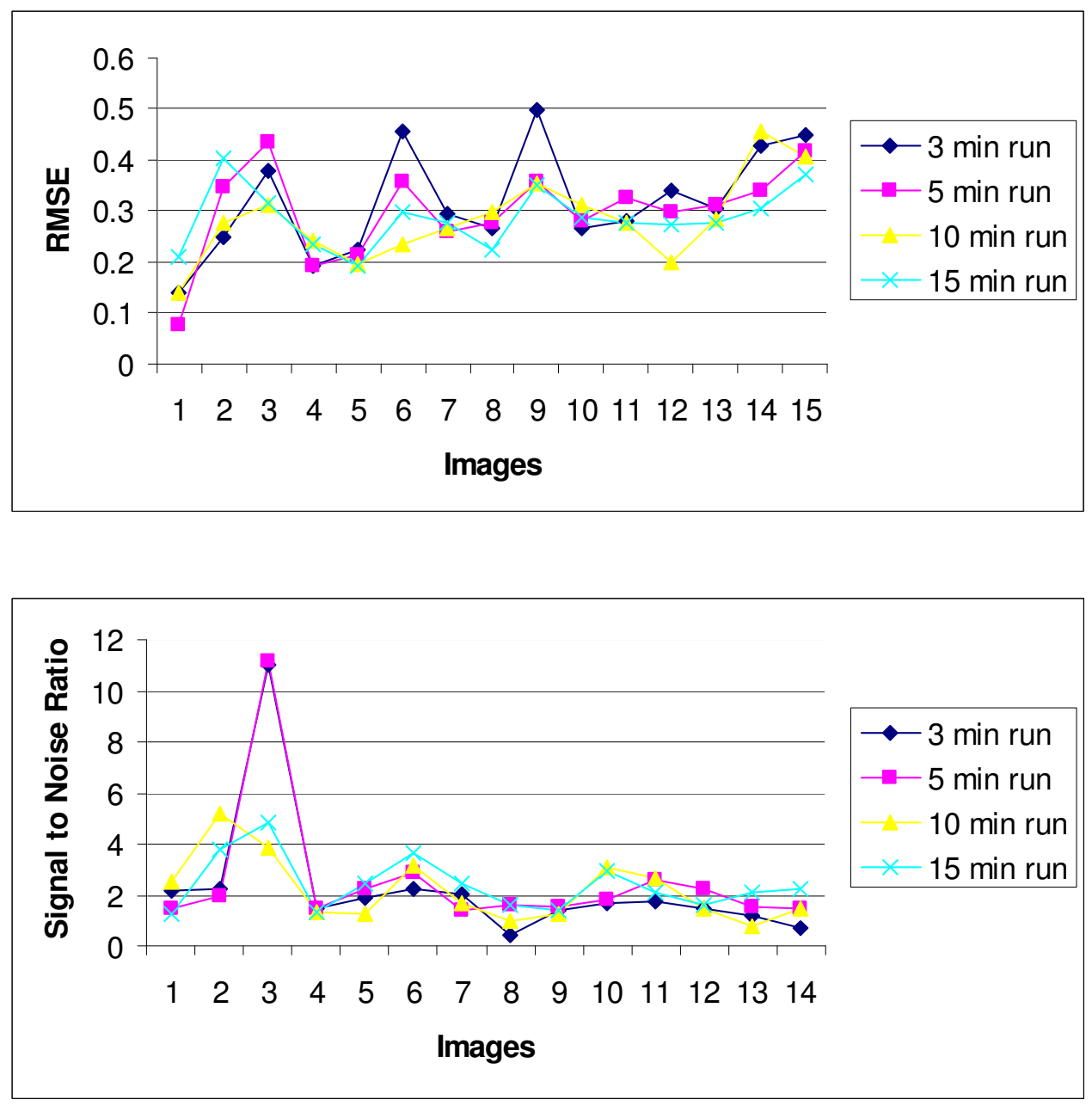

Figure 5.3 Plots of RMSE and SNR for different termination criterion

From the plots above we infer that the lowest error between the subject and reference images is in more test cases with a termination criterion of a ten minute time limit. With ten time limit on the registration process the graphs depict low RMSE and high Signal to Noise ratio compared with 3, 5 and 15 minute time limit. 


\subsection{TESTING WITH KNOWN TRANSFORMATION PARAMETERS}

In the second method of testing, we adopt a known set of parameters for testing our approach. We generate some random affine parameters of a, b, c, d, e, f. We apply these known parameters on the query image. The transformed image obtained is considered as the reference image in this testing method. We then implement our multi resolution genetic algorithm based registration on the new reference and query images. The resultant transformation parameters are noted. These are the estimated transformation parameters.

We then compare the values of the estimated and known transformation parameters. The query dental region of interest is mapped accurately to reference dental region of interest when the transformation set produced by the approach is similar to that of the known transformation parameters.

We have conducted the testing with more than 100 dental image pairs with a specified time limit of ten minutes for each dental image pair. 55 of these test regions of interest showed percentage error between the actual and estimated transformation parameters below $20 \%$. We calculated the Mean square error and the Root Mean square error values between known transformation parameters and the resultant estimated transformation parameters. We plot the average MSE and RMSE values followed by SNR values for registered pairs. The plots shown in this chapter are for 55 test pairs. The diagram showing the process flow of testing using known transformation parameters is as follows: 


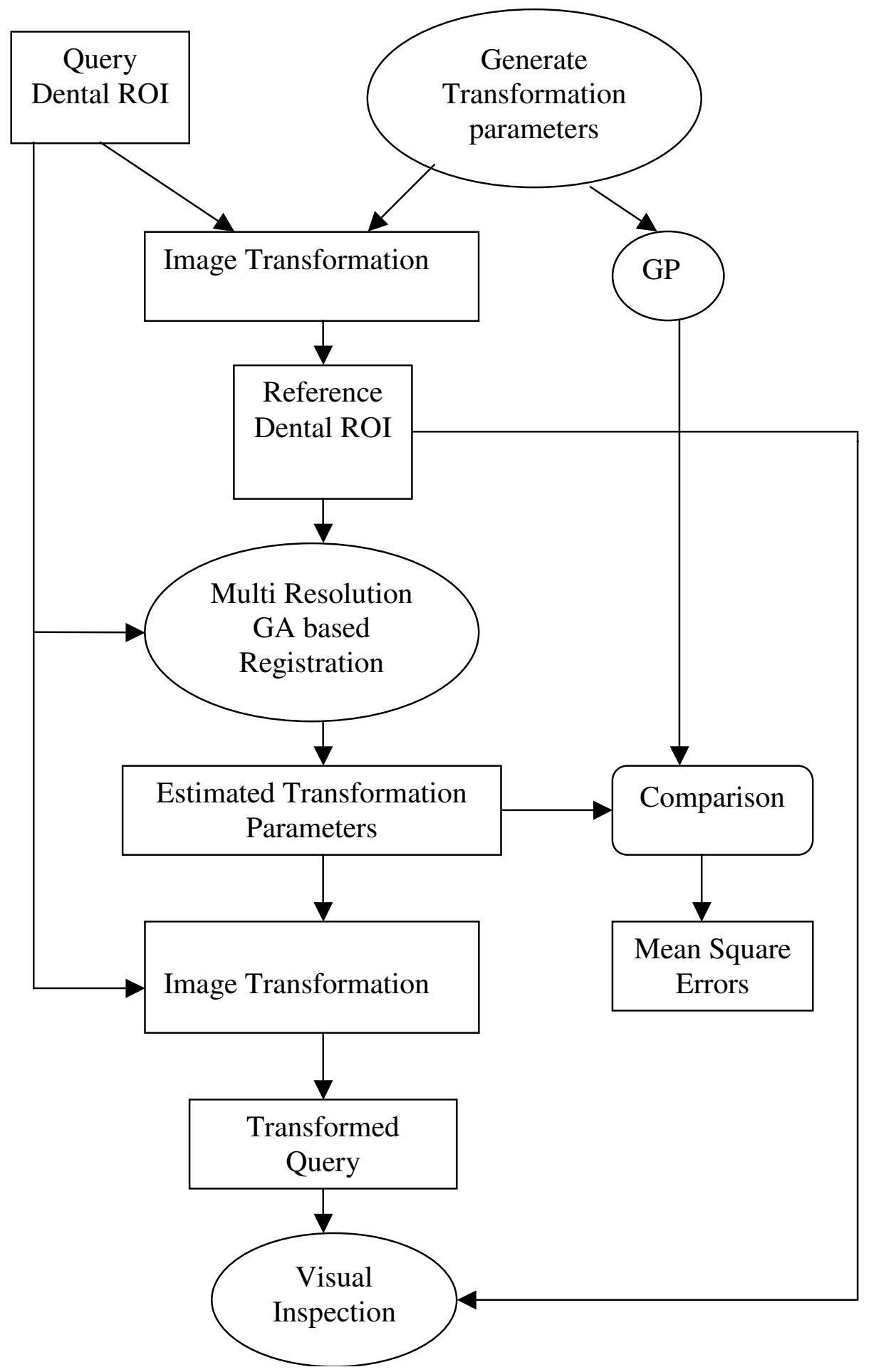

Figure 5.4 Block diagram for testing using known transformation 
Figures below depict the plot of RMSE and Signal to Noise ratios for registered ROI's.
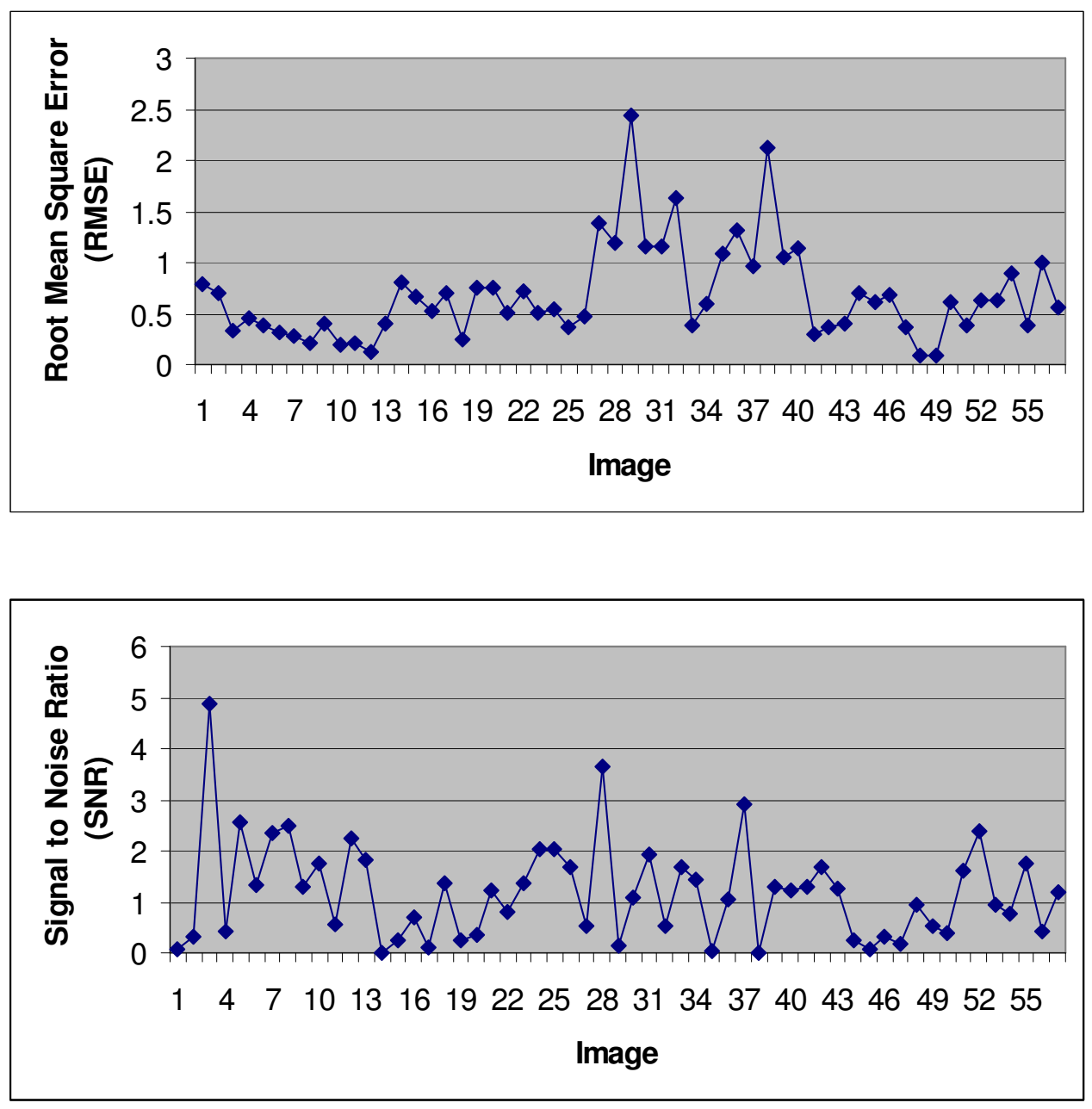

Figure 5.5 Plots of RMSE and SNR for Dental ROI's

The low RMSE values between the subject and reference images from the plots in figure 5.4 indicate good performance in accuracy level. Test cases that are heavily misaligned show increase in the RMSE values (e.g. images from 25 to 40). Signal to Noise ratio is a measure to determine the extent of noise present in images. The low Signal to noise ratio in most cases indicates high noise distributed over the entire test dental images. 
These are the plots for the termination criterion using 3, 5, 10 and 15 minute run times for the RMSE and SNR between the images with the known parameter testing.
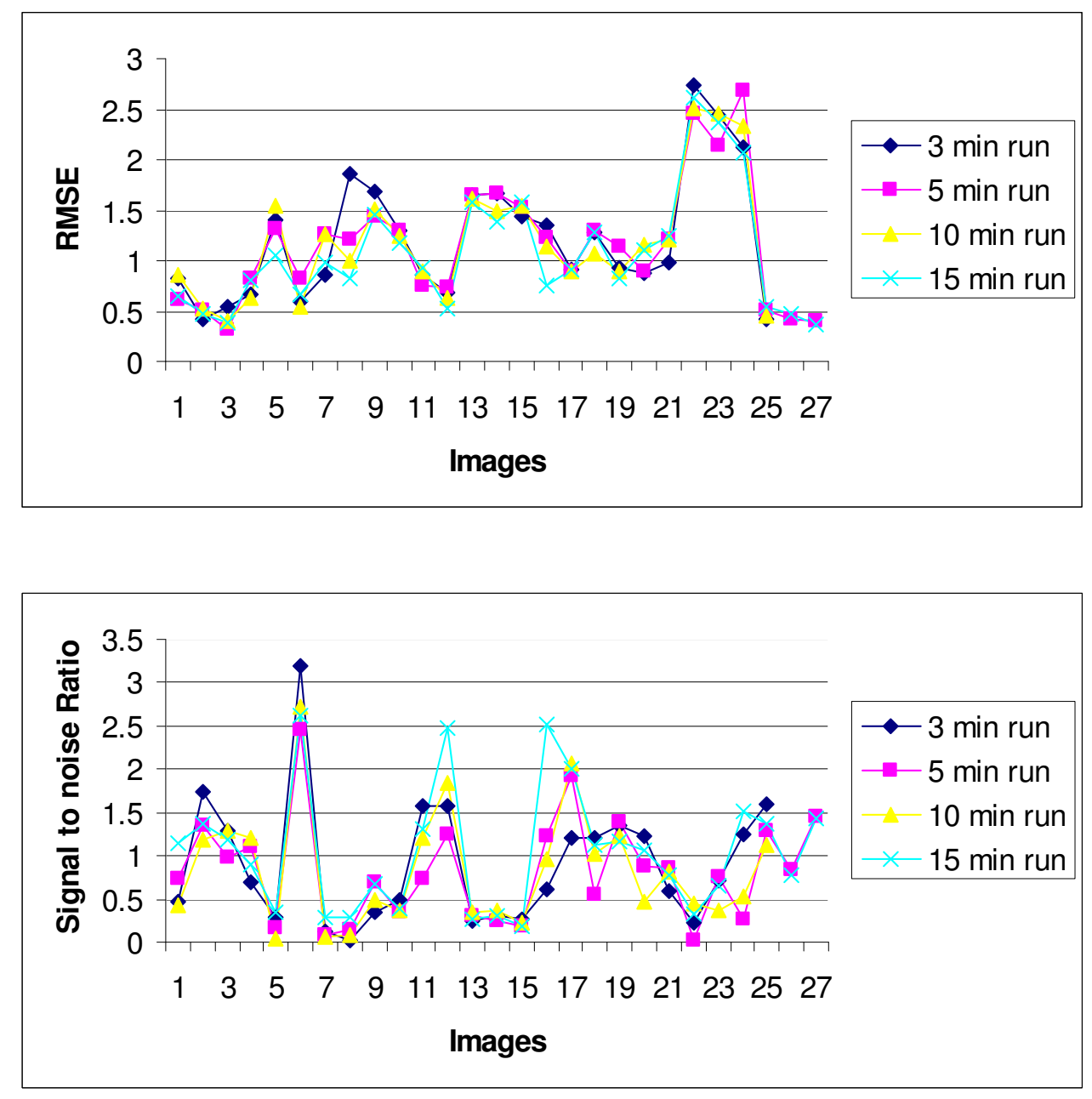

Figure 5.6 Plots of RMSE and SNR for different termination criterion

From the plots above we infer that the RMSE and SNR between the subject and reference images is the same in most test cases with different termination criterion. 
In the figure below we plot the Average MSE and RMSE for Dental ROI's
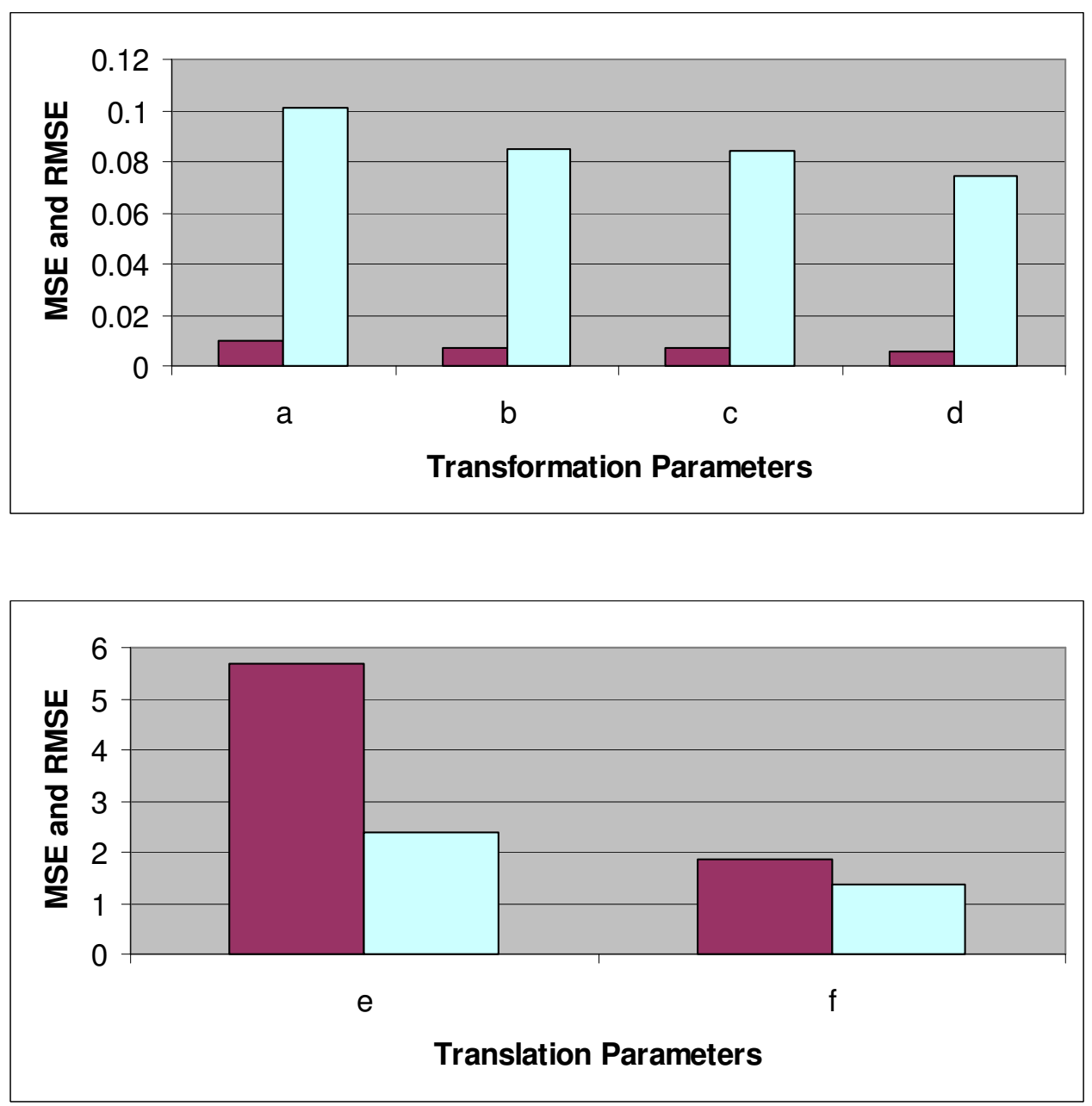

Figure 5.7 Plots of Average MSE and RMSE for Transformation Parameters

The above plots depict the MSE and RMSE values between the known and the estimated transformation parameters for 55 dental test pairs. The low RMSE values indicate good performance and accuracy level.

From the results obtained we calculated the relative percentage errors between known and the estimated transformation parameters. The Following figure shows plots of Average Relative Percentage errors. 

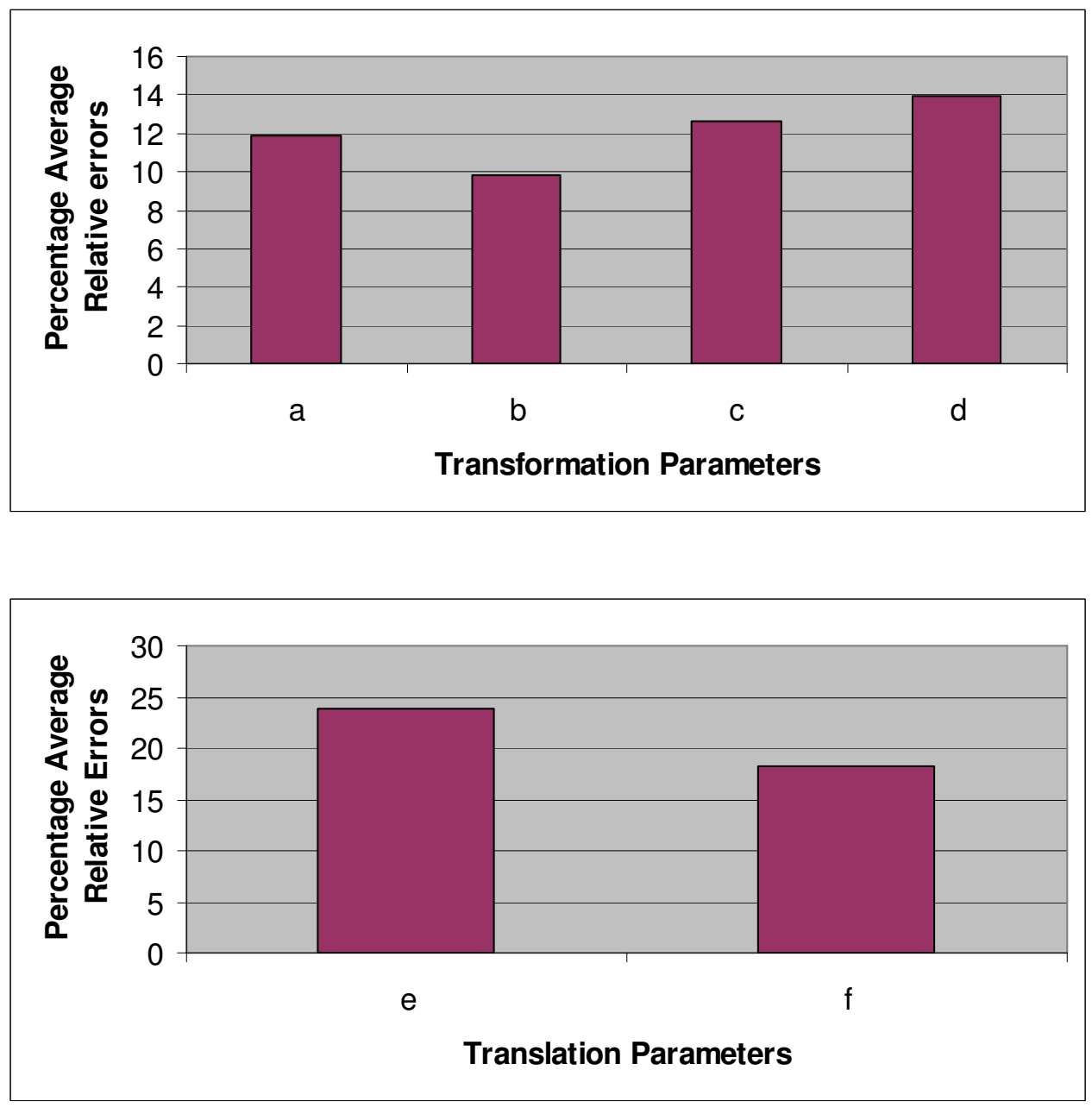

Figure 5.8 Plots of Average Relative Percentage errors for Transformation parameters

From results we observed that the query dental region of interest mapped accurately to dental region of interest in test cases that contain slight misalignments using this approach. The relative percentage errors between the known and estimated transformation parameters was below $20 \%$ for registered dental image pairs using this technique with a termination criteria of a ten minute time limit. We could achieve this error on the dental image pairs when the subject and reference images differed by slight distortions, containing less noise and degradations. The technique produces reasonable error upto 18 degree misalignment with respect to rotation and a translation upto 16 pixels. 


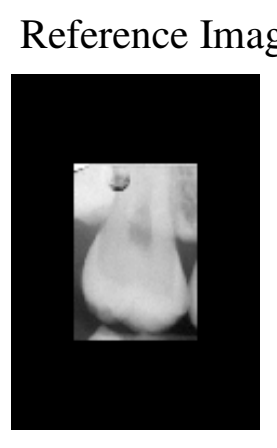

Subject Image

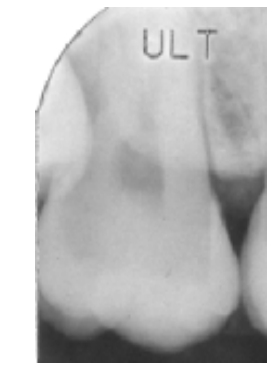

Registered output
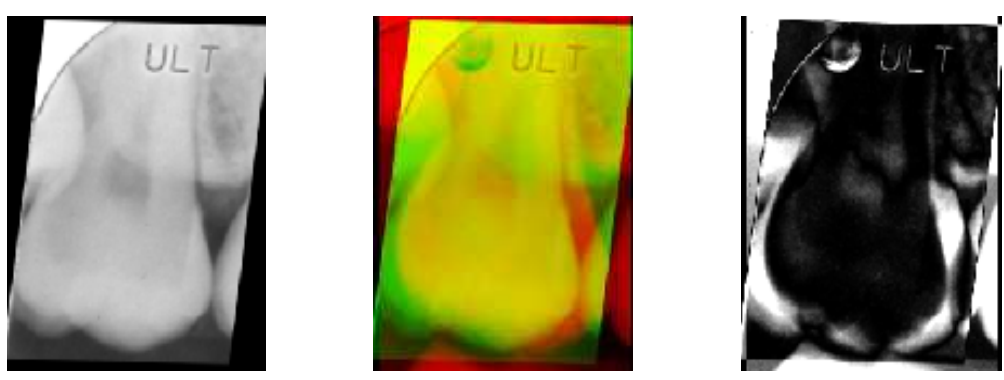

Figure 5.9 Example using Unknown Transformation Parameters

Figure above shows the example of registered dental cases with testing using unknown transformation parameters. By Inspection we find that the transformation parameters showed good accuracy. 

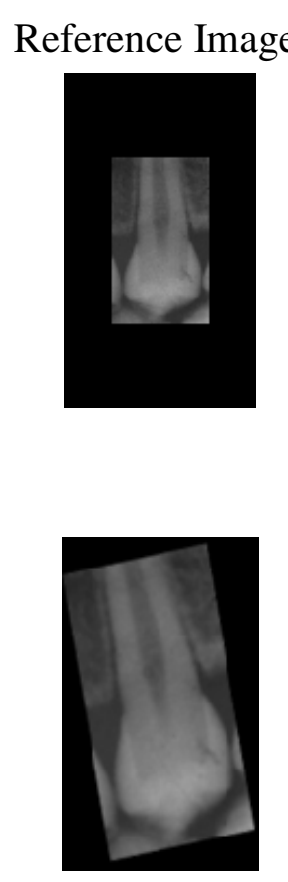

Subject Image

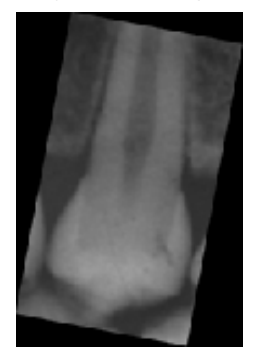

\section{Registered output}
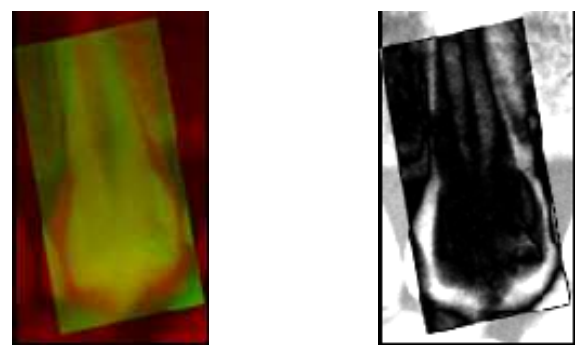

Figure 5.10 Example using known Transformation Parameters

Figure above shows an example of registered dental cases with testing using known transformation parameters. The error between known and the estimated transformations was below $20 \%$ in this case due to slight misalignment between the subject and reference image. 


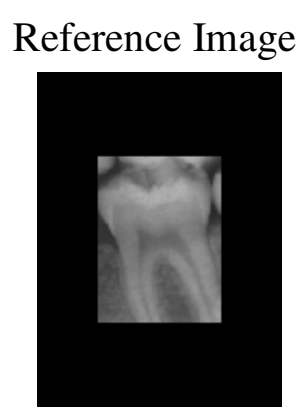

Subject Image

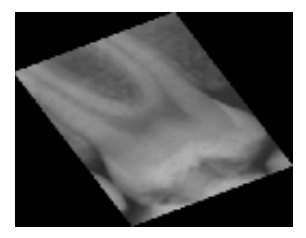

Registered output
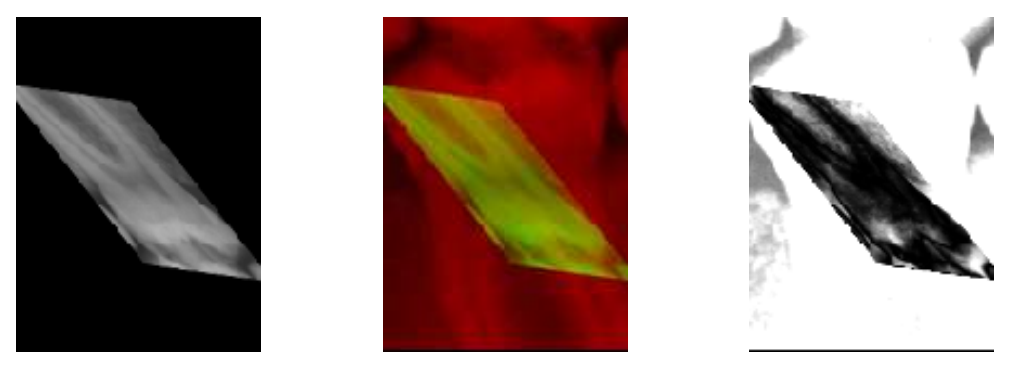

Figure 5.11 Unregistered Pairs due to high variation between subject and reference

Figure above depicts an example of unregistered dental cases due to heavy misalignment between the subject and reference image. 

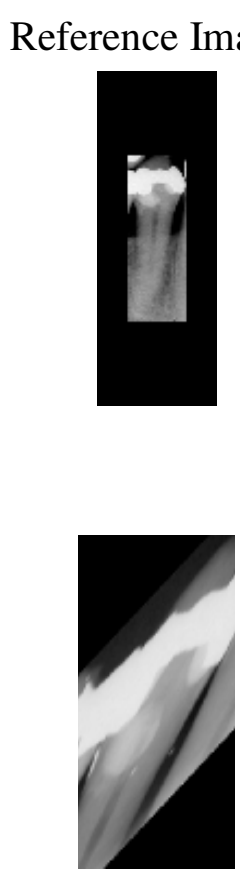

Subject Image

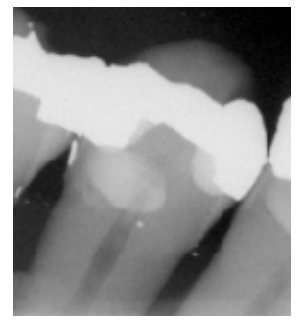

Registered output
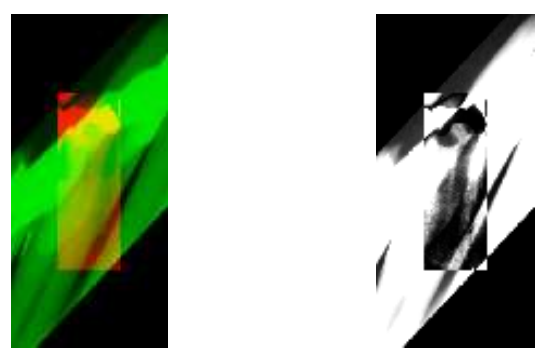

Figure 5.12 Unregistered Pairs due to Partial Visibility of teeth and heavy misalignment

Figure above shows an example of registered dental cases due to Partial visibility of teeth and heavy misalignment between the subject and reference images. 


\section{CHAPTER 6}

\section{CONCLUSIONS}

In this chapter we present a brief conclusion of the dental image registration elucidating our goals and achievements.

This thesis presented dental image registration technique, one of the preprocessing steps under image comparison component before the final matching and decision making. The main objective was to achieve automatic registration of subject dental regions of interest with the corresponding reference dental regions of interest.

We have investigated and implemented registration using multi resolution techniques namely image sub sampling and wavelet decomposition. Multi resolution techniques helped in speeding up registration process by limiting the registration process to useful search data. Edges were employed as features to be matched. We adopted a Oriented Hausdorff Similarity measure that yields a similarity measure between the subject image and the corresponding reference dental region of interest. The similarity algorithm is proved to be robust to severe noise, degradations and outliers present in the images.

An affine transformation for transforming the subject image was selected that incorporates changes due translation, rotation, scaling and shearing of the subject image. It was useful in capturing some distortions in images. The genetic algorithm search strategy generated better solutions with increasing generations and image resolutions. Thus a reduction in search space along with accurate registration parameters was achieved for some dental cases.

The proposed approach gives reasonably accurate transformation parameters to test cases that are slightly misaligned. Unclear edge detection due to noisy dental images resulted in obtaining inaccurate similarity measures. The method could not capture some complex distortions. Registration using multi resolutions along with genetic search strategy though 
reduced the search data and search space turned to be time consuming. Therefore we have adopted a termination criterion with a ten minute limit irrespective of the resolution level in which the registration is being carried out.

From the testing results we conclude that using this image registration technique the relative error on the transformation parameters was below $20 \%$ for dental test cases where the subject and the reference images contain slight distortions, containing less noise and degradations. These variations in the dental test cases are due to differences in lighting conditions, view points, time instances and sensor characteristics. The technique produces reasonable error for up to 18 degree misalignment with respect to rotation and a translation for up to 16 pixels. The technique yields large errors for dental images that are heavily distorted and contain considerable noise and degradations. Further research is required as outlined in the next chapter. 


\section{CHAPTER 7}

\section{FUTURE WORK}

In this chapter we predict the scope of future work for the proposed multi-resolution dental image registration based on genetic algorithm.

Image registration plays a vital role in most Forensic identification systems. Since research in various new technologies in Biometrics and Forensic Science is fast escalating, we emphasize on a wide range of improvements for registration techniques. As such there is a lot of scope for improvements in the proposed dental image registration algorithm.

This multi-resolution dental image registration based on genetic algorithm developed for Automated Dental Identification system effectively registered some dental ROI pairs. We obtained errors below $20 \%$ error on slightly misaligned test images and unexpected errors on most dental images. Thus our research left us with some areas of the image registration that are yet to be explored and investigated.

Therefore, we propose some issues of our registration technique that provide scope for future implementation. As discussed in the previous chapters, we have adopted edges as features for registration, and adopted Sobel Edge detection technique. Better edge detection techniques can be investigated that provide clear edges even at lower resolution of the image. Noise present in images is another factor that can be considered for future work on edge detection methods

We have applied affine transformations on the subject dental image, and estimated bounds on the transformation parameters for dental image registration. The PM dental regions of interest on which we have tested our approach contain many complex distortions that caused misalignments. An extension of transformation from Affine to Projective or Euclidean could be suggested. 
We have investigated and applied image sub sampling and wavelet decomposition techniques for obtaining various resolutions of the dental ROI's. An attempt to capture vital edge information of the blurred and partly occluded dental images in the lowest resolutions may result in accurate transformation parameters.

Further refinement in the parameters of genetic algorithm search strategy using multipopulations may yield speedy generation of final registration parameters. 


\section{REFERENCES}

[Ammar00] H.H Ammar, Y.Tao, "Fingerprint registration using genetic algorithms", Application-Specific Systems and Software Engineering Technology Proceedings. 3rd IEEE Symposium on, 24-25 (March 2000), Pages: 148 -154.

[Bajscy89] R. Bajcsy, S. Mosaic, "Multiresolution elastic matching", Computer Vision, Graphics and Image Processing 46 (1989) 1-21.

[Ballard81] D. H. Ballard, "Generalizing the Hough transform to detect arbitrary shapes." Pattern Recognition, v. 13, n. 2, pp. 111-122, 1981.

[Barnea72] D.I. Barnea, H.F. Silverman, “A class of algorithms for fast digital image registration", IEEE Transactions on Computing 21 (1972) 179-186.

[Barrow77] H.G. Barrow, J.M. Tanenbaum, R.C. Bolles, H.C.Wolf, "Parametric Correspondence and chamfer matching: Two new techniques for image matching". Proceedings of the Fifth International Joint Conference on Artificial Intelligence, Cambridge. (1977)

[Brown92] L.G. Brown, "A survey of image registration techniques", ACM Computing Surveys 24 (1992).

[Bruel02] Thomas M. Breuel, "A Comparison of Search Strategies for Geometric Branch and Bound Algorithms", (2002).

[Castro87] E.D. Castro, C. Morandi, "Registration of translated and rotated images using finite Fourier transform", IEEE Transactions on Pattern Analysis and Machine Intelligence 9 (1987) 700-703.

[Chalermwat99] P. Chalermwat, "High Performance Automatic Image Registration for Remote Sensing”, PhD. Thesis. - GMU, (Fall 2001).

[Fitz84] J.M. Fitzpatrick, J.J. Grefensette and D. Van Gucht, "Image Registration by Genetic Search", Proceedings of IEEE South East Conference, pp 460-464.

[Gatbx] A.J. Chipperfield, P.J. Flemming "The Matlab Genetic Algorithm Toolbox", User's Guide, Version 1.2.

[Goshtasby85] A. Goshtasby, G.C. Stockman, "Point pattern matching using convex hull edges", IEEE Transactions on Systems, Man and Cybernetics 15 (1985) 631-637. 
[Gonza02] R.C. Gonzalez and R.E. Woods, "Digital Image Processing", 2nd ed., Prentice Hall, (2002).

[Huttenlocher93] D.P. Huttenlocher, G.A. Klanderman, W.J. Rucklidge, "Comparing images using the Hausdorff distance", IEEE Transactions on Pattern Analysis and Machine Intelligence 15 (1993) 850-863.

[Hero00] Bing Ma, Alfred Hero, John Gorman, "Image Registration with Minimum Spanning Tree Algorithm”, ICIP (2000).

[Jain86] Jain A., "Fundamentals of Digital Image Processing", Prentice-Hall, (1986).

[Jain99] K. Jain, Rudd Bolle, Sharath Pankanti, "Biometrics: Personal Identification in Networked Society", Kluwer Academic Publishers, (15 January 1999).

[Kneöaurek00] Karin Kneöaurek, Marija Ivanovic, Josef Machac, David A. Weber, "Medical image registration". Euro physics News (2000)

Vol. 31 No. 4

[Millwala04] Millwala. Z., "A Dual Stage Approach to Dental Image Registration", Masters Thesis. Department of Electrical and Computer Engineering - WVU, December (2004).

[Kugli75] C.D. Kuglin and D.C. Hines, "The Phase Correlation Image Alignment Method", Proc. IEEE 1975 Int. Conf. Cybernetics and Society, (September 1975), pp163-165.

[Nassar03] Nassar D.E., Dr. Hany Ammar, "A Prototype Automatic Dental Identification System" (ADIS), Department of Electrical and Computer Science Engineering - WVU. (March 2003).

[Nassar05] Nassar D.E., Mythili Ogirala, Zainab Millwala, D. Adjeroh and H.Ammar, "Bounds on Image Alignment Parameters under Affine Transformation", Submitted to ICASSP 2005.

[Ostuni93] J. Ostuni, E. Fisher, P.V. Stelt and S. Dunn, "Registration of dental radiographs using projective geometry", Dentomaxillofacial Radiology, Vol 22, Issue 4 199-203.

[Rangarajan99] A. Rangarajan, H. Chui, J.S. Duncan, "Rigid point feature registration using mutual information", Medical Image Analysis 4 (1999) 1-17

[Ritter99] N. Ritter, R. Owens, J. Cooper, R.H. Eikelboom, P.P. van Saarloos, "Registration of stereo and temporal images of the retina", IEEE 
Transactions on Medical Imaging 18 (1999) 404-418.

[RM03] West Virginia University Research Corporation-West Virginia University (2003).

[Samad04] F. Samadzadegan, F. Bashizadeh, M. Hahn, P. Ramzi, "Automatic Registration of Dental Radiograms", Geo-Imagery Bridging Continents, Commission 5, XX ISPRS Congress, 12-23 (July 2004) Istanbul, Turkey.

[Sato95] J. Sato, R. Cipolla, "Image registration using multi-scale texture Moments", Image and Vision Computing 13 (1995) 341-353

[Shekhar99] C. Shekhar, V. Govindu, R. Chellapa, "Multisensor image registration by feature consensus", Pattern Recognition 32 (1999) 39-52.

[Sim01] Dong-Gyu Sim, Rae-Hong Park, "Two Dimensional Object Alignment Based on the Robust Oriented Hausdorff Similarity Measure", IEEE Transactions on Image Processing Vol.10, No 3. (March 2001).

[Stockman82] G. Stockman, S. Kopstein, S. Benett, "Matching images to models for registration and object detection via clustering", IEEE Transactions on Pattern Analysis and Machine Intelligence 4 (1982) 229-241.

[Thevenaz98] P. Thevenaz, M. Unser, "An efficient mutual information optimizer for multiresolution image registration", Proceedings of the IEEE International Conference on Image Processing ICIP'98, Chicago, IL, (1998), pp. 833-837.

[Venot84] A.Venot, J.F. Lebruchec, and J.C. Roucayrol, "A New Class of Similarity Measures for Robust Image Registration", Computer Vision, Graphics and Image Processing 28,(1984), pp 176-184.

[Yoon00] D. C. Yoon, "A new method for the automated alignment of dental radiographs for digital subtraction radiography", Dentomaxillofacial Radiology, Vol 29, Issue 1 11-19, (2000).

[Zheng93] Q. Zheng, R. Chellapa, "A computational vision approach to image Registration”, IEEE Transactions on Image Processing (1993)

311-325. 